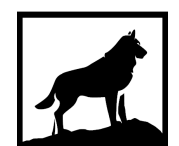

Michigan Technological

1 8 8 5 University
Michigan Technological University Digital Commons @ Michigan Tech

2017

A WIRELESS, PASSIVE SENSOR FOR MEASURING TEMPERATURE AT ORTHOPEDIC IMPLANT SITES FOR EARLY DIAGNOSIS OF INFECTIONS

Salil Sidharthan Karipott

Michigan Technological University, sskaripo@mtu.edu

Copyright 2017 Salil Sidharthan Karipott

Recommended Citation

Karipott, Salil Sidharthan, "A WIRELESS, PASSIVE SENSOR FOR MEASURING TEMPERATURE AT

ORTHOPEDIC IMPLANT SITES FOR EARLY DIAGNOSIS OF INFECTIONS", Open Access Master's Thesis, Michigan Technological University, 2017.

https://doi.org/10.37099/mtu.dc.etdr/342

Follow this and additional works at: https://digitalcommons.mtu.edu/etdr

Part of the Biomedical Commons, and the Biomedical Devices and Instrumentation Commons 


\title{
A WIRELESS, PASSIVE SENSOR FOR MEASURING TEMPERATURE AT ORTHOPEDIC IMPLANT SITES FOR EARLY DIAGNOSIS OF INFECTIONS
}

\section{By}

Salil Sidharthan Karipott

\begin{abstract}
A THESIS
Submitted in partial fulfillment of the requirements for the degree of MASTER OF SCIENCE

In Biomedical Engineering
\end{abstract}

MICHIGAN TECHNOLOGICAL UNIVERSITY

2017

(C)2017 Salil Sidharthan Karipott 
This thesis has been approved in partial fulfillment of the requirements for the Degree of MASTER OF SCIENCE in Biomedical Engineering.

Department of Biomedical Engineering

\begin{abstract}
Thesis Advisor:
Committee Member:
\end{abstract}

Committee Member:

Department Chair:
Dr. Keat Ghee Ong

Dr. Jingfeng Jiang

Dr. Smitha Rao

Dr. Sean J. Kirkpatrick 


\section{Table of Contents}

List of Figures............................................................................................................................. vi

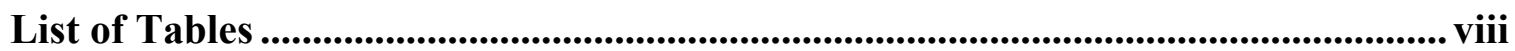

Acknowledgement .................................................................................................................. ix

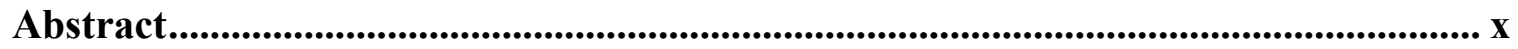

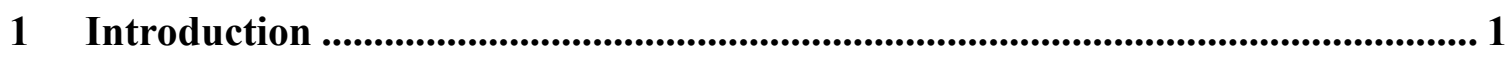

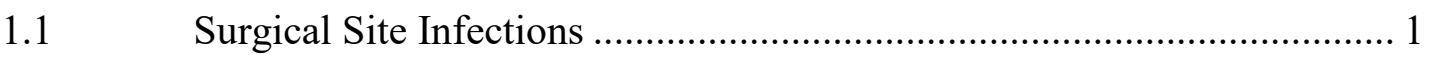

1.2 Infections Related to Orthopedic Surgeries ........................................ 2

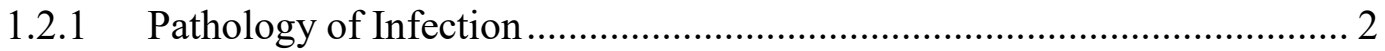

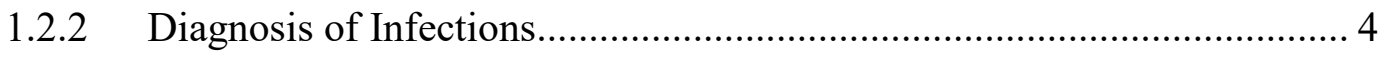

1.2.3 Treatment of Infection in Orthopedic Surgeries .................................... 5

$1.3 \quad$ Ligament Reconstruction Surgeries ....................................................... 6

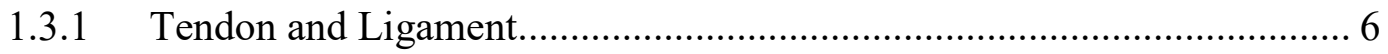

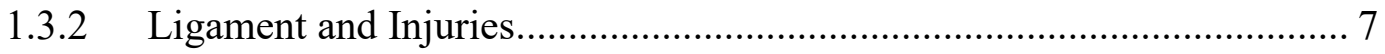

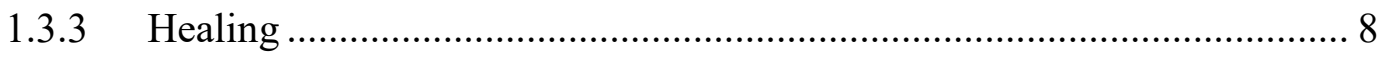

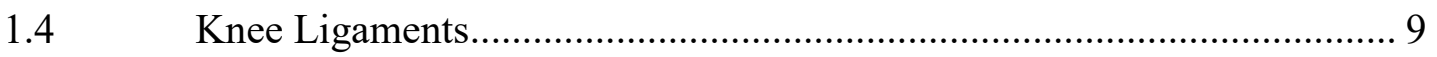

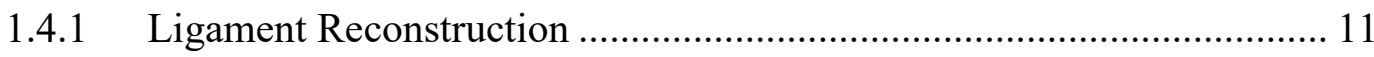

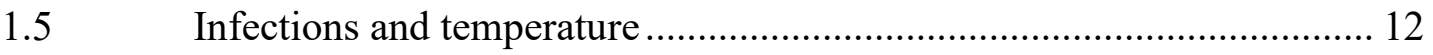

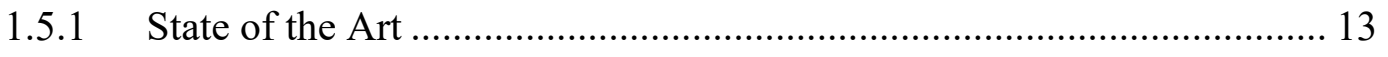




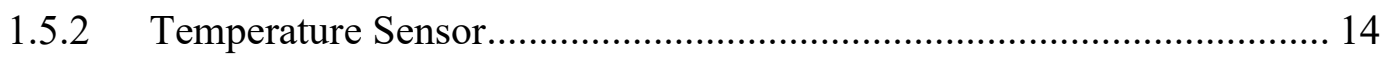

1.6 Wireless and Passive Temperature Sensor............................................ 15

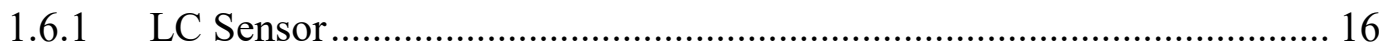

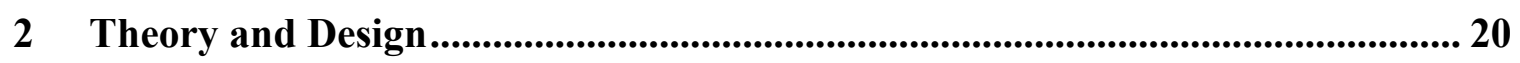

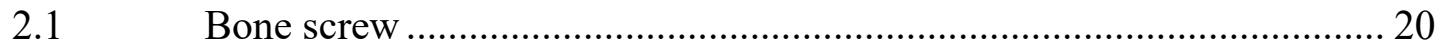

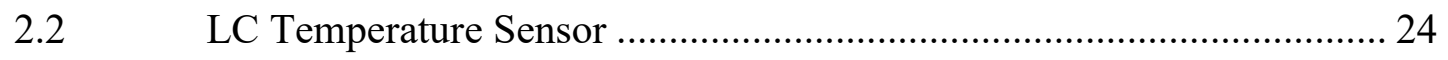

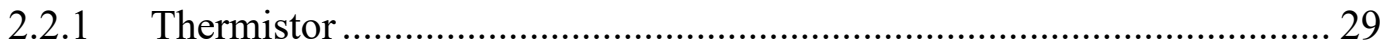

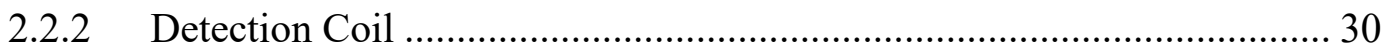

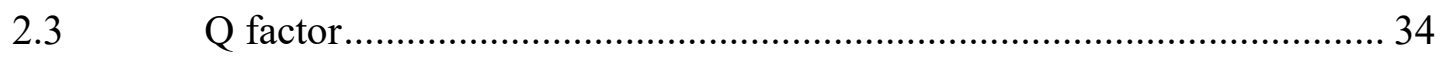

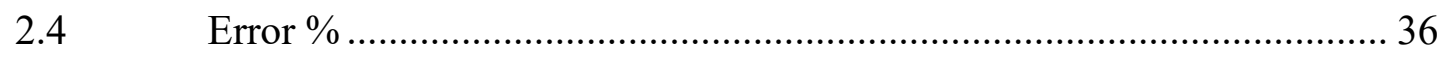

$3 \quad$ Experiment and Results .............................................................................................. 37

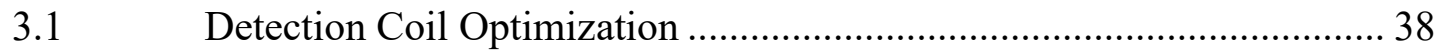

3.1.1 Distance between Sensor and Detection Coil ........................................ 38

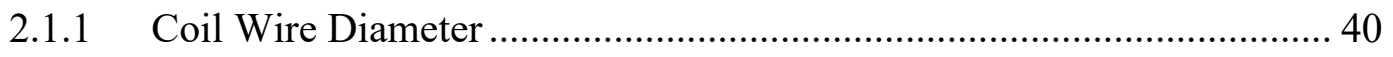

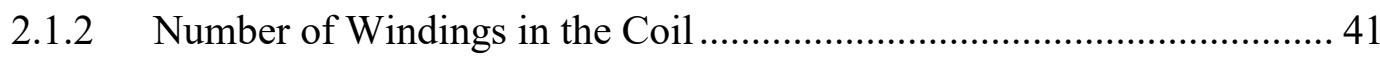

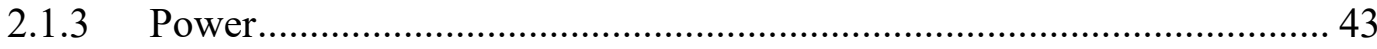

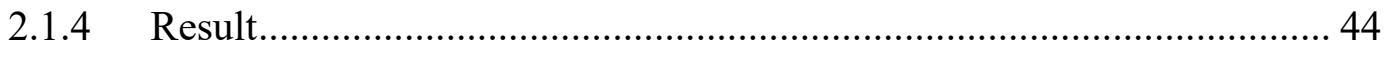

3.2 Sensor Optimization ........................................................................ 45

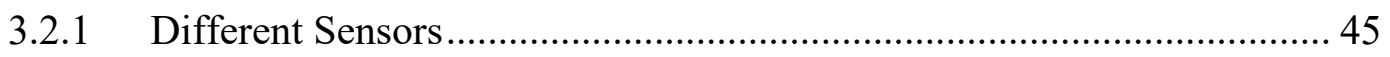

3.3 Relative Position Sensors and Detection Coil ........................................ 47 iv 
Sensor Performance................................................................... 49

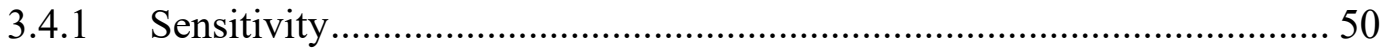

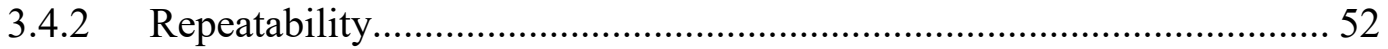

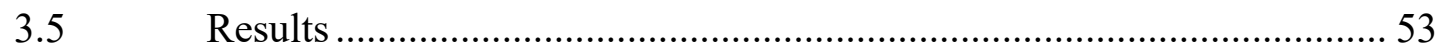

Sensor performance in actual tissue ................................................ 55

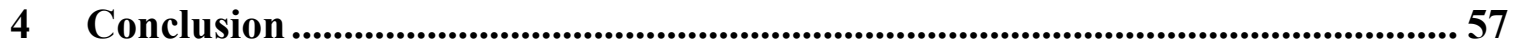

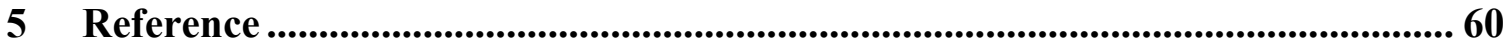




\section{List of Figures}

Figure 1.1 Illustration of interference screw placement for MCL reconstruction. ........... 11

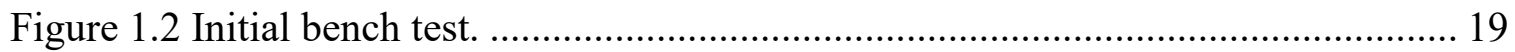

Figure 2.1 Illustration of the designed sensor....................................................... 21

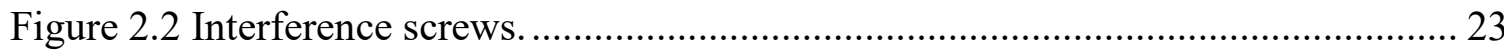

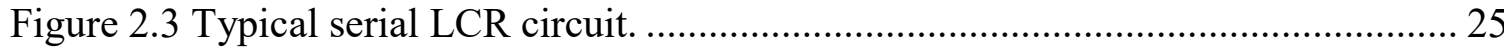

Figure 2.4 Layout of SMD thermistor and Capacitor .............................................. 28

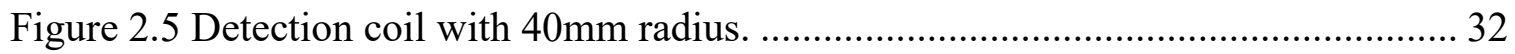

Figure 2.6 Magnetic field variation with size of coil $(30 \mathrm{~mm}, 40 \mathrm{~mm}$ and $65 \mathrm{~mm})$ at $30 \mathrm{~mm}$

Figure 2.7 Frequency response plot for sensor $0 \mathrm{~mm}$ from of $30 \mathrm{~mm}$ detection coil ........ 34

Figure 2.8 Frequency response plot for sensor $20 \mathrm{~mm}$ from of $65 \mathrm{~mm}$ detection coil ..... 35

Figure 2.9 Bandwidth, resonant frequency and noise for a typical frequency response. . 35

Figure 3.1 Sensor response for change in distance of separation of sensor and coil and

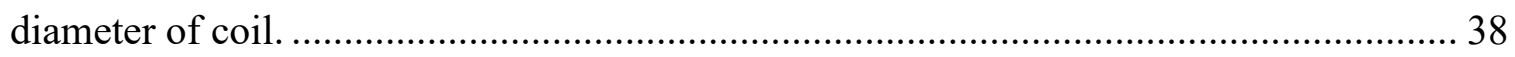

Figure 3.2 Sensor response for change in coil diameter at $0 \mathrm{~mm}$ separation................... 39

Figure 3.3 Sensor response for different diameters of the wire................................ 40

Figure 3.4 Sensor response with different number of windings. .............................. 42

Figure 3.5 Sensor response with change in power applied to the detection coil. ............ 43

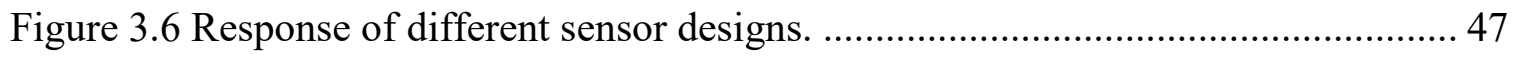

Figure 3.7 Sensor response with change in perpendicular distance between the cylindrical

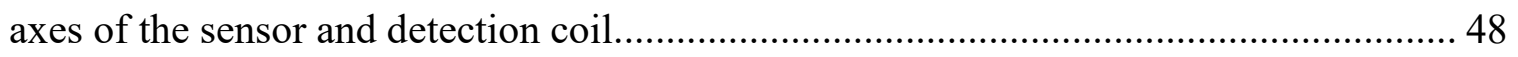


Figure 3.8 Sensor response with change in angle between cylindrical axes of the sensor and

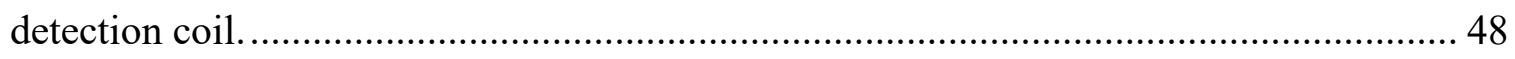

Figure 3.9 Q factor versus Thermistor resistance ............................................... 51

Figure $3.10 \mathrm{Q}$ factor versus Temperature, ............................................................ 51

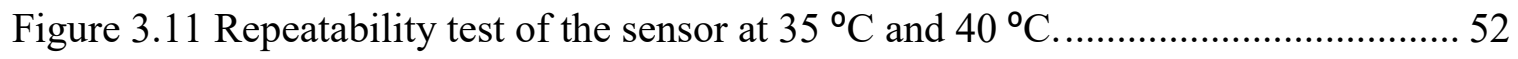

Figure 3.12 Temperature measured by sensor versus true temperature measured by the

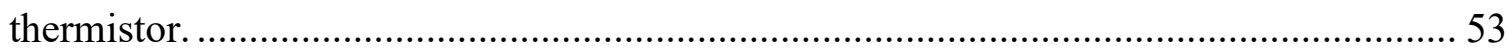

Figure 3.13 Difference in sensor performance with materials.................................... 56 


\section{List of Tables}

Table 2.1 Different interference screw sizes designed and their corresponding sensors

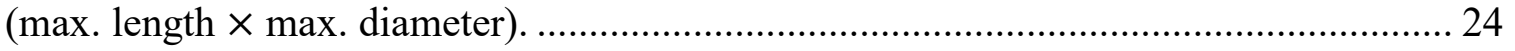

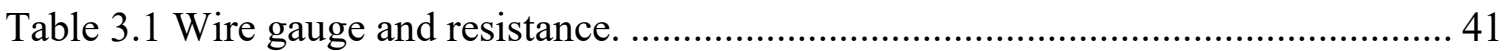

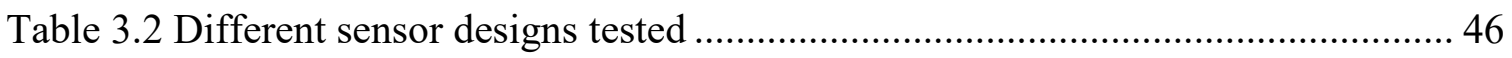




\section{Acknowledgement}

I would like to sincerely thank my adviser Dr. Keat Ghee Ong for his constant support and guidance during the course of completion of this project. His immense knowledge and work ethics has helped shape my knowledge and research skills.

I would also like to convey my gratitude to my committee members, Dr. Jingfeng Jiang and Dr. Smitha Rao for imparting their time and knowledge for the completion of this project. I would like to thank my friends in Houghton and back home for their support and motivation. I also would to express my gratitude to Kri Nga Dow and John.

Finally, I would like to thank everyone in my family for believing in dreams. 


\begin{abstract}
Sensorized implants with embedded wireless, passive temperature sensors were developed for early detection of implant-associated infections. The operation principle of the sensor is based on the hypothesis that infections can lead to an increase in local temperature prior to the rise of body temperature. The sensor was an inductive capacitive (LC) circuit that has been used for monitoring of different parameters wirelessly, often in difficult to access environments. The sensor was fabricated on to an interference screw, which is used for tendon and ligament reconstruction surgeries. In this project, a sensorized interference screw was designed and fabricated by accommodating an LC sensor. Different designs of sensors and detection coils were made and tested for optimal performance.
\end{abstract}

Infection at the site of an orthopedic implant is a serious challenge in the field of orthopedic surgery. These infections can lead to adverse health and economic burdens for the patients. The rate of failure of implants due to infections was around $2 \%$ to $2.4 \%$ during 2001-2009 period and rising. The treatment cost for orthopedic-associated infections has increased to 566 million USD in 2009 and is projected to 1.62 billion USD by 2020 . Several techniques are used to evaluate infections, including X-rays radiography, bone scans, and lab blood tests, but primarily it is based on swelling and increased pain at the site of infection. Several studies have shown relations between temperature and infections, they focused on surface tissue layers and to our knowledge, there have been few similar studies in deeper layers. The goal of this project is to develop a device that can operate within deeper tissues. 


\section{Introduction}

\subsection{Surgical Site Infections}

Surgical Site Infections (SSI's) are infections induced by pathogens at the site of a surgery. SSIs adversely impact the patient in the postoperative period, augmenting treatments and delaying recovery [1] which predispose patient comfort and economic expenditure negatively. US Centers for Disease Control and Prevention (CDC) classifies SSI into 3 different types [2]:

i. Superficial Incisional SSI: Infections to the subcutaneous tissue at the site of incision for the surgery.

ii. Deep Soft Tissue SSI: Infections of fascia and muscle tissue at the site

iii. Organ/space SSI: Infection to body tissue other than subcutaneous and muscle tissue.

SSIs show common symptoms like redness, delay in healing, fever, pain, tenderness, or swelling. Internally, pus is formed as a part of the immune response. Pus is a collection of dead granulocytes along with bacteria and tissue debris. Pus can collect within or in between tissue layers to form abscess, and this occurs mostly in organ/space SSIs. The abscess is revealed only during re-opening of the wound site or using specialized X-ray imaging. Organ/space SSIs are more common for orthopedic implants since the tissue is already immunologically compromised with the introduction of a foreign body. According to CDC data for 2002 [3] SSI contribute to $20 \%$ of all Hospital Associated Infections (HAI). Early detection is the key in reducing complication that may arise due to the infection. There are studies showing correlation between infection in surgeries and the 
local temperature at the implant site [4-8]. This project focuses on producing a sensor system to monitor temperature at the site of an orthopedic implant based on the hypothesis that infection at the site of implant causes an increase in local temperature

\subsection{Infections Related to Orthopedic Surgeries}

Orthopedic surgeries contribute a lot to the above-mentioned SSI statistics due to the substantial number of sports injuries and accidents which almost always lead to orthopedic surgeries. Even though the rate of infections related to orthopedic surgeries remain low, the actual number of cases of orthopedic SSIs are increasing due to the rise in the number of orthopedic surgeries. A survey from 2001-2009 has concluded that the infection rates for hip and knee replacements ranged from $2.0 \%$ to $2.4 \%$, and the infection rate continues to increase over time [9]. The economic expense related to the infections remain high due to the need of infection related treatments, revision surgeries, rehospitalizations and prolonged hospital stays. During the period 2001-2009, the annual cost for treating knee and hip replacement infections increased from \$320 million to $\$ 566$ million, and is projected to increase to $\$ 1.62$ billion by 2020 [9]. Other surveys have also shown similar results, indicating the implant-associated infection to increase at rates at around $1-2 \%[10]$.

\subsubsection{Pathology of Infection}

Infection related to orthopedic surgeries mainly involving implants have been studied in detail in recent years. This has given important insights into pathogenesis of 
infection, and improved diagnostic and treatment methods. Sendi et al., [11] classifies orthopedic infections based on inoculation,

i. Exogenous infections: This type of infections usually occur during the early postoperative period when pathogens enter the site directly during the surgery.

ii. Hematogenous infections: The infections that occur due to pathogens from blood and it may manifest as early as right after the surgery or in later stages than the exogenous infections, and even show up years after the actual surgery.

Zimmer et al., [12] classifies infections according to the time at which they occurs as: early, delayed and late. Early infections occur within 2 months of the surgery and these are mostly exogenous. Delayed infections can occur up to 2 years after the surgery. These infections can be either exogenous or hematogenous. Late infections are those which diagnosed after 2 year of the surgery and are mostly hematogenous when there are no initially diagnosed infections.

The infection causing pathogen are mostly bacteria such as_Staphylococcus aureus, Escherichia coli and Propionibacterium acnes. Among the microorganisms, the most common are coagulase-negative Staphylococci (30-43\%), S. aureus (12-23\%), Streptococci (9-10\%), Enterococci (3-7\%), gram negative Bacilli (3-6\%) and anaerobes (2-4\%). In addition, $10-11 \%$ infections were found to be polymicrobial infections [13, 14].

The mechanism of infection is explained by a biofilm formation, which consists of surface adhering bacteria and extracellular matrix produced by them. There are several 
different factors that contribute towards the progression of infection, including microorganisms' resistance to host response and impaired host response. Biofilms adhering to the implant surface have microcolonies of one or more species of microorganisms. They can proliferate into colonies with considerably larger numbers, thus increasing their chances to be resistant to host response. This resistance is accounted by the quorum-sensing system that regulates the production and release of various virulence factors [15]. Similar biofilms are also observed in dental plaques and endocarditis. Impaired host response is another important factor in orthopedic implant infections. Frustrated phagocytosis, a functional defect in granulocytes (White Blood Cells responsible for inflammatory response) leads to degranulation, impaired ingestion, and super peroxide production. Also, biofilms trigger massive host response which causes more tissue damage near the site of infection. Biofilms produce chronic inflammations, which have shown to cause mutations in the microorganism. There are also chances of induction of antibiotic resistance due to this mutation even though most mutations can reduce the microorganism's proliferation [16].

\subsubsection{Diagnosis of Infections}

Initial diagnosis of the implant-related infection is similar to other bacterial infections, which is based on symptoms like pain, redness, and swelling at the site of the implant. Medical diagnostic methods include C-reactive protein (CRP) test, which account for the inflammation in the body. Since the CRP levels are high in the blood for up to 14 days post-surgery, a lower CRP can be used to rule out infections, while a persistent high 
value in routine test can only suggest a possibility of infections [17]. Since CRP is measured from blood, an infection elsewhere in the body can also relate to high CRP values. Radiography is used for imaging the site of orthopedic implant. It can be used to diagnose bone loss and implant loosening. Furthermore, MRI is usually not preferred for certain implants due to metallic artifacts. Ultrasound is used for imaging inflammation related swelling near the site and can also be used as evidence guide for carrying out aspirations to collect samples for histology and microbiology, which can then yield better results than the other diagnostic methods [18].

It should be noted that while there are a wide variety of techniques for diagnosis of infections, there is still a lack of decisive and sensitive method for diagnosis at early stages of infection. Although aspirations are sensitive and specific, they are only performed in a case where there is considerable evidence of infections from imaging methods, externally visible swelling and redness or extreme patient discomfort.

\subsubsection{Treatment of Infection in Orthopedic Surgeries}

Treatment of infections require a combination of different techniques and the patient conditions and comorbidities. Most treatment methods involve antibiotics along with surgical interventions [19]. For some patients with several other comorbidities, surgeries are typically not carried out and the infections are treated conservatively with antibiotics alone. Otherwise, surgical interventions including joint removals, revision surgeries, amputations to name a few, are executed in different infection progression stages. 


\subsection{Ligament Reconstruction Surgeries}

A major portion of orthopedics related injuries and surgeries are contributed by ligament injuries. Patients with ligament injuries are mostly young athletes with ligament tears in lower proximity joints such as knees and ankles $[20,21]$ related to contact sports such as football, rugby, basketball.

\subsubsection{Tendon and Ligament}

Tendon and ligament are soft collagenous connective tissues which functionally connect and support muscles and bones. Ligaments connect bones to bones and tendons connect muscles to bones (tendons also attach muscles to other organs as in the case of eyeballs). They play a major role in the musculoskeletal system, facilitating relative motion of bones and muscles. Both tendon and ligament have a similar hierarchical structure. The mechanical properties of tendons and ligaments are similar to that collagen, which constitutes about $86 \%$ dry weight in tendons and is just slightly lesser percentage in ligaments [22]. The ligament tears can be explained by mechanical events [23, 24]. For example, without considering viscoelastic properties of ligaments, tendons and ligaments exhibit a non-linear elastic behavior. There are three regions in the stress strain graph of ligaments: toe region, linear region, and, yield and fracture region. The ligaments usually work in the toe region and sometimes in between toe and linear region of the stress strain graph. Since the ligaments are considered elastic, the deformation in this regions is reversible. When stress increase beyond the linear region there is yield and fracture region. 
Once yielding occurs, it is not reversible and leads to fractures. Effects of loading with time can be explained by the viscoelastic behavior of ligaments. Creeping is another mechanical event that can explain the fracture of ligaments [25]. Creep is defined as the elongation under a constant load. With increase in time, this elongation can eventually lead to fracture. These basic mechanically events can be used to explain sports injuries due to over use and strain of the joint. Major tendon and ligament injuries are observed in Achilles tendon, knee ligament tears and rotator cuff injuries [26-28].

\subsubsection{Ligament and Injuries}

The terms ligaments and tendons are both alternately used due to their similarities in structure and function but the biochemical ratio and functions are different. Ligament are more metabolically active than tendons. Ligaments are mostly found in joints, where they are attached to two different bones across a joint to facilitate relative motions between joints. They also act as position sensor in joints $[29,30]$ and provide stability to joints.

Ligaments are the focus in this project since there is high number of ligament injuries and related reconstruction surgeries, and interference screw are mostly used in ligament surgeries. The system can be replicated for use in tendons as well with small dimensional and design variations. Furthermore, ligaments tears in knee injury contribute a major portion to orthopedic injuries and often require surgical intervention. A majority of these injuries are sports related. Mechanically these injuries have been classified on the basis of the mechanism of injury [31].

- Contact or direct trauma 
- Dynamic loading

- Repetitive overuse

- Structural vulnerability

- Poor flexibility

- Muscle imbalance and

- Rapid growth

Mechanical loading characteristics such as magnitude, location, direction, duration, frequency, variability, and rate of loading defines the principal of sport related injuries $[32]$.

\subsubsection{Healing}

Healing is a slow process, especially when it comes to ligament injuries. There are several factors that determine the time required to heal, such as the extent of ligament tear, and the position of ligament. For example, Medial Collateral Ligament (MCL) in the knee heals much faster than the Anterior Cruciate Ligament (ACL) [33]. ACL is exposed to joint fluids in the knee, and the tear causes the ends to be in contact with the fluid which does not favor healing. Even after the natural healing process the tissue does not regain it natural mechanical properties for a long time. The general biological response after a ligament injury can be divided into three phases [34]:

- Bleeding and inflammation

- Active repair of rupture and

- Remodeling 
During the initial period, and right after the injury proper care should be taken to reduce bleeding and edema. This decreases the chance of scar tissue from being hypertrophic, stiff and painful.

During the repair phase, movement should be restricted at least in the initial 3 weeks after the injury as this was found to affect the orientation of collagen fibrils after healing. After this period, motion will help to increase the strength in ligament. Repair phase is marked by collagen synthesis which peaks at 3 weeks [35].

The remodeling phase can be from anytime between 6 weeks after injury to up to 1 year. The scar tissue that has already formed matures into normal tissue. The phase is marked by increased levels of collagen and normal water content. In a long term study by $\mathrm{Ng}$ (1996) et al., [36], it was found that a goat ACL was able to recover most of its tensile strength in 1 year. After 3 years, the strength even surpassed that of normal ACL tissue. However, the mechanical properties like Young's Modulus did not return to normal even after 3 years.

\subsection{Knee Ligaments}

Human knee is one of the most important joints in human body. There are two articulations in knee: the tibiofemoral joint and patellofemoral joint. It is the largest joint in the body, connecting the thigh to leg. Knee joint is a type of modified hinge joint, which allows extension and flexion with very limited internal and external rotation. The knee joint constitutes of 3 functional compartments [37]: 
- Patellofemoral Articulation, formed by the patellar grove in front of the femur and the patella which slides through the grove.

- Medial Tibiofemoral Articulation.

- Lateral Tibiofemoral Articulation, both connecting the tibia and femur in between which synovial fluid is encapsulated inside the synovial membrane.

The ligaments of the knee are classified into two on the basis of their position: intracapsular which lie within the joint itself, and extracapsular which towards the outer surface of the joint.

i. Intracapsular ligaments: These ligaments control most of the knee movements. It is made up of two ligaments; the Anterior Cruciate Ligament (ACL) which connects the lateral condyle of femur to the anterior intercondylar area, and the Posterior Cruciate Ligament (PCL) which connects medial condyle of femur to the posterior intercondylar area.

ii. Extracapsular ligaments: It is made up of two ligaments on the medial and lateral side of the knee. Medial Collateral Ligament (MCL) connects the femur and tibia on the medial side and Lateral Collateral Ligament (LCL) connects femur to fibula.

Ligament injuries or tear occur when the knee sustains irregular motion or direct force impact on the ligaments. For example, twisting and bending of knee often lead to torn $\mathrm{ACL}$, direct force impact causes PCL tear, and LCL and MCL tears are usually caused by lateral or medial bending forces. 


\subsubsection{Ligament Reconstruction}

Ligament reconstruction surgeries are only carried out in case of total ligament rupture. Partial ruptures are often treated with a combination of rest and exercise. This constrain in surgery is due to the high risks of reconstruction failure. Reconstruction can cause infections, graft rejection or improper rehabilitation. In a study conducted in 1999 [38], it was found that $20-25 \%$ of ACL and $60 \%$ of PCL reconstruction surgeries are not successful. Similar failure rates for ACL surgeries were noted in study published in 2011 [39].

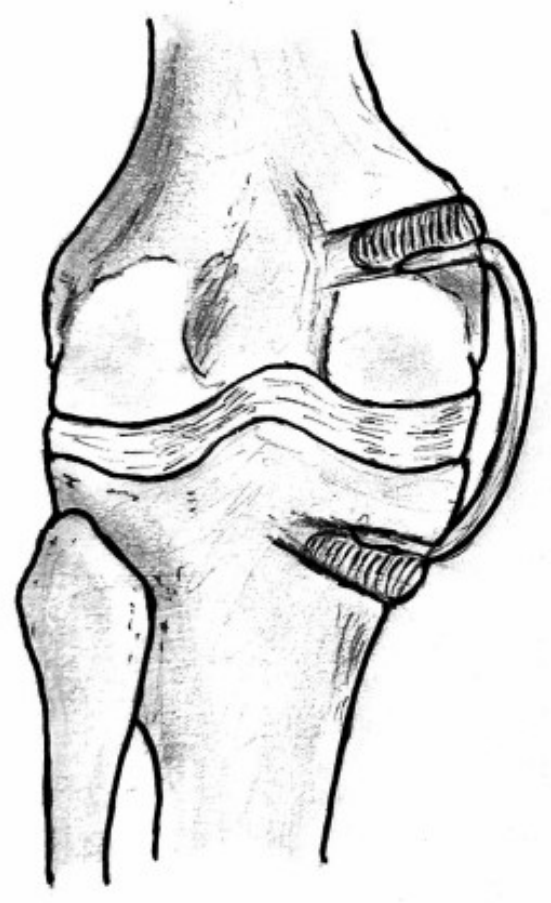

(a)

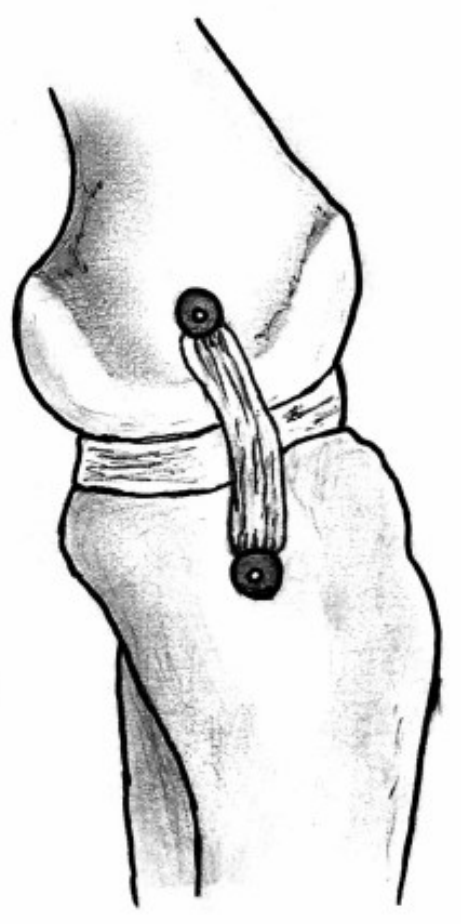

(b)

Figure 1.1 Illustration of interference screw placement for MCL reconstruction.

(a) The posterior view shows screws through the medial epicondyle and (b) medial view showing the ligament graft stretching over the joint 
The first ACL graft was reported by Hey Groves [40]. Majority of the reconstructions are done in young patients, who are usually athletes, and people with chronic knee instabilities. Allografts (grafts of tendon or ligament form others, usually cadavers) or autografts (graft is taken from other parts of the patient body) are usually used for reconstruction. Commonly used grafts are bone patellar tendon autograft, allograft and semitendinosus autograft. The grafts are fixed with the help of different anchoring techniques like interference screws, anchors or combination of two or more techniques.

Reconstruction surgeries are carried out with great care. Surgeries are not carried out in inflammatory phase, which is typically 3-4 weeks after the ligament tear, due to the high risk of arthrofibrosis. Inflammatory condition also slows down healing and graft remodeling [41]. Surgeon's precision and type of graft determine the success of reconstruction surgery. Graft remodeling is a long process and it can take years for the graft to attain normal mechanical strength of ligament.

\subsection{Infections and temperature}

Temperature has been associated with wound healing and infection for a long time. And there have been several studies showing infection related temperature rise. These studies have used surface temperature at the site of implantation as measure to identify infection. Although the mechanism behind infection and temperature change is not fully understood, studies have shown that temperature measurement specificity in identifying infections $[4-6,8,42]$. 
Possible physiological interactions that can cause changes in temperature included the immune response and bacterial proliferation. The immune response triggers different physiological reactions such as localized inflammation, oedema, hematoma and callus formation. As a part of the pro-inflammatory response, increased blood flow to the region contributes to a high concentration of heat at the site. The metabolic activity of the bacteria can also contribute to increasing temperature. With or without infections, temperature also changes after surgeries. Experiments based on a bone defect model in goats has been shown that there can be an initial drop in temperature at the site of a surgery attributed to damaged blood vessels [43]. Therefore, to use temperature to diagnose infections, further studies need to be done to better understand temperature variation and regulating factors.

\subsubsection{State of the Art}

Several studies were conducted to understand how temperature varies at the site of infection. There have been several studies on the basis of thermographic imaging, using infrared thermometers to monitor infections on the surface and deep layers of tissue. With recent advances in technology, cheaper and more efficient thermographic and telethermographic imaging is now possible. Studies have shown effective use of thermography in septic knee prosthesis, healing in knee and hip prosthesis and several different infection diagnostics $[4-6,43,44]$.

Thermography as explained by Romano et al., (2011) [45] had a good specificity and sensitivity. But the thermographic study was done on patient already having joint pain at the site of surgery, which suggests that the infection should be significant enough to be 
detected on the outer tissue layers. Therefore, although this experiment only shows correlation between temperature and infections, it provides the basis of our hypothesis to use temperature at the site of implant for early diagnosis of infections. We hypothesize that if a temperature sensor is placed directly at the implant site, it will be possible to detect it even before thermographic detection is possible.

A study by Epari et al., [43] shows temperature measurements in sheep during 4 post-operative days. The study shows that initially the temperature was lower than the normal body temperature in tibia osteotomy. The temperature reached to the normal body temperature in 4 days. Epari et al., also points out in the study about the lack of sensor system that can effectively make long duration monitoring because the study used catheter type sensors. The disadvantages pointed out in the work include:

- The environment in which the catheter is placed may not relate directly to the environment around the fracture because the inclusion of blood from the fracture may have triggered blood flow and hematoma around the sensors.

- Using a sensor can also affect the healing process and long term study was not possible.

\subsubsection{Temperature Sensor}

The most important part of any temperature sensor is achieving a good thermal coupling with the object being measured. Thermal coupling can be achieved by transfer of thermal energy between the object and the sensor. The sensor converts this thermal energy into an electrical signal. Heating up and cooling down the sensor involves transfer of 
thermal energy, which can induce errors in measurements [46]. Infrared thermometers use radiation for thermal energy transfer. This non-contact type temperature measurement can introduce variability and error induced by the operator and environmental factors. A catheter type sensors also have similar limitations like limited access to the site and error induced due to presence of catheter at the site [43]. A sensorized implant can reduce these errors by measuring the temperature around an implant since the energy transfer is due to conduction and not by any external factor. An ideal sensor for this application should have the following features:

- Size: Small size to reduce error due to thermal energy transfer and enabling easy integration to currently available orthopedic devices.

- Wireless: Sensor system should be wireless to minimize infection that can be related to wires or catheters placed internally or externally in the body.

- Passive: An internally perpetual system is ideal for long term monitoring. The sensor should not have any power supply placed inside the body, as it should be externally powered or capable of energy harvesting.

\subsection{Wireless and Passive Temperature Sensor}

A new sensor was developed to monitor infections in orthopedic implants to help predict the start of infections. The sensor can lead to better understanding of the relation between temperature and infection and the mechanism behind it. Even though infections and temperature rise are usually correlated, there are very little studies done in this field. Majority of the studies focus on infections and ulcers near to the skin [4, 7, 42]. We would 
like to pursue a similar approach on deeper tissue layers by using a wireless system. The fact that infections can arise for up to 2 years after surgery is a great deal of concern [12], and the wireless temperature sensor system may provide a solution for this challenge. A passive system which does not need power or that can be powered externally will help to achieve long term studies even after several years. This project focuses on producing a device that can act as a research tool, and in turn can be commercialized to be an actual product.

In this project, temperature sensors were embedded in interference bone screws. These bone screws are widely used in ligament and tendon reconstruction surgeries, and associated to a large number of orthopedic surgeries. The small size of the inductivecapacitive resonant circuit sensor is ideal for this applications because the interference screws are small in size themselves. Being wireless and passive, the sensor does not interfere in the implantation process. Also, the sensor will not interface with the body during or after the implantation.

\subsubsection{LC Sensor}

LC sensors or Inductive-Capacitive Resonant Circuit Sensors are tank circuits with a specific resonant frequency that is determined by the inductance and capacitance in the circuit [47-51]. They are wireless and passive sensors which are powered externally through an excitation/detection coil. They can change their resonant characteristics such as resonant frequency and quality factor depending on the inductance, capacitance and 
resistance values in the circuit. LC sensors come with different designs. For this sensor, the main components are:

- Inductive winding: It is responsible for the inductive coupling between the sensor and the detection coil, which allows wireless transmission of sensor information to an external device.

- Capacitor: It regulates the resonant frequency of the sensor.

- Thermistor: This is the sensing element for this sensor design, responsible for transduction of temperature. The temperature change causes the resistance of the thermistor to vary. This resistance change is measured as a change in the quality factor of the sensor.

The sensor is interrogated by suppling an alternating voltage to the detection coil. The alternating voltage induces a magnetic field and the magnetic field in turn produces a voltage in the sensors inductive coil. This is the magnetic coupling between the sensor and the coil. The sensor is interrogated at different frequencies. At the resonant condition of the sensor, the total impedance of the LC circuit reduces to its resistance. In an ideal LC circuit without resistive loss the impedance should be zero. However, for this sensor design, the impedance in the sensor is a function thermistor resistance. When the sensor couples with the coil, the impedance of the sensor is inversely reflected in the impedance of the coil. This means that at resonance, the measured impedance at the detection coil is at its peak, and an increase in the resistance of the sensor decreases the magnitude of the measured impedance. Since the resistance of the sensor depends on the temperature, impedance measurement of the coil can thus be used to measure temperature. 
The LC sensor and passive RFID (Radio Frequency Identification) [49, 52-55] sensors are based on the same principle of electromagnetic induction. RFID is being used widely in consumer products as wireless tag [55]. However, RFIDs are susceptible to component failure and limited range [55]. The inclusion of miniature circuit also increases fabrication cost. The main advantage of LC sensors over RFID is the ease of fabrication and low cost. Passive RFID sensors need to have AC to DC converters on board the sensors. The factors determining the resolution of sensor also depends on the onboard components of the RFID circuit. There are fewer components in LC sensors as compared to RFID sensors.

Ong et al., [52] demonstrated the use of LC based sensor in monitoring growth of bacteria. In the study, bacteria growth was measured using a planar LC circuit with interdigital capacitor and planar inductive coils. The permittivity of the capacitor was used as a measure of bacterial growth. The planar LC sensor was placed below a culture of bacteria. As bacteria proliferated in a culture, the permittivity experienced by the interdigital capacitors in the sensor increased. Ong et al., [52] also demonstrated the use of similar planar sensors for monitoring temperature, humidity and pressure. Several other printed resonant circuits have been developed to measure parameters such as strain, $\mathrm{pH}$ and relative humidity [49]. A planar LC circuit is difficult to be manufactured into small size and without affecting the sensor's performance. However, the same electronic circuit can be replicated using a solenoid inductor and a surface mount capacitor to match the shape and size of interference screw. Ong et al., [50] sensor work on the basis of capacitance change, which in turn varies the resonant frequency. In this senor, the changing 
parameter is the resistance in the LC circuit, which is measured in terms of the quality factor of the resonant curve. This was studied in an initial bench top model shown in Figure 1.2 .
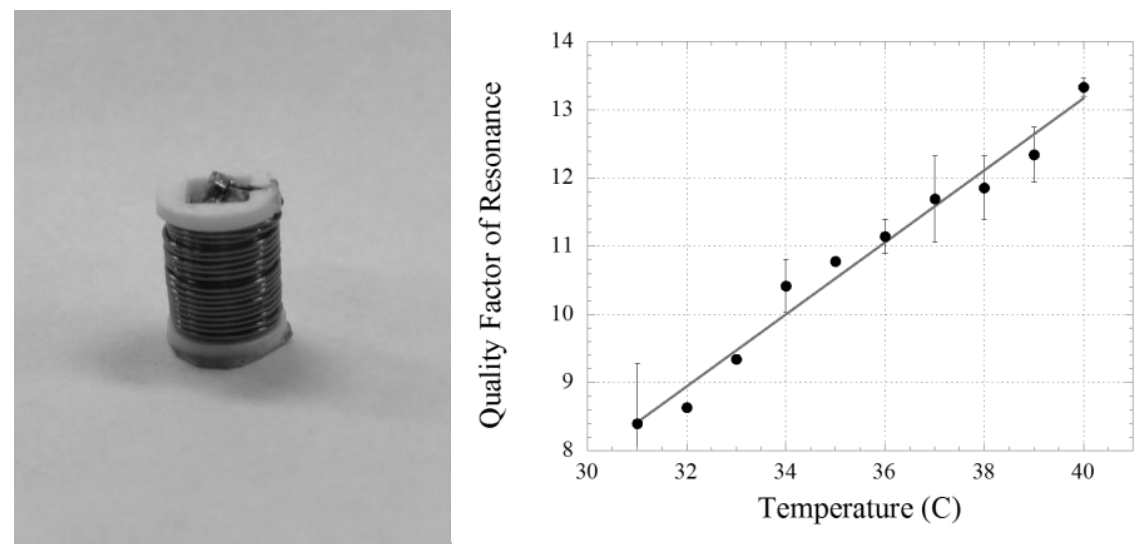

Figure 1.2 Initial bench test.

(a) Initial test model and (b) test results showing the linear variation of quality factor in terms of changes in temperature. 


\section{Theory and Design}

\subsection{Bone screw}

Interference bone screws are used for fixing tendons and ligaments [56, 57]. These screws were chosen for the study due to the high incidence of tendon and ligament injuries in recent years [9]. Currently, these reconstruction surgeries are conducted using different materials including stainless steel, titanium and non-metal polymers such as PEEK, PLA and PGA, with mixture of BCP (Biphasic calcium phosphate, which contains b-TCP and HA). The polymer screw was selected instead of metals in the project to reduce electromagnetic shielding effect caused by metallic objects around the sensor. The design was based upon a commercially available bone screw from Arthrex ${ }^{\circledR}$ (Tenodesis Screw, BioComposite, $8 \mathrm{~mm} \times 12 \mathrm{~mm}-\mathrm{AR}-1680 \mathrm{BC})$. Interference screws are manufactured by several other companies including Smith \& Nephew, Zimmer and Stryker. These screws basically differ in their thread pitch, screw driver types, dimensions and polymer material, depending on the intended site of application. Arthrex interference screw was used because of the ease to design and prototype, and also its popularity. 


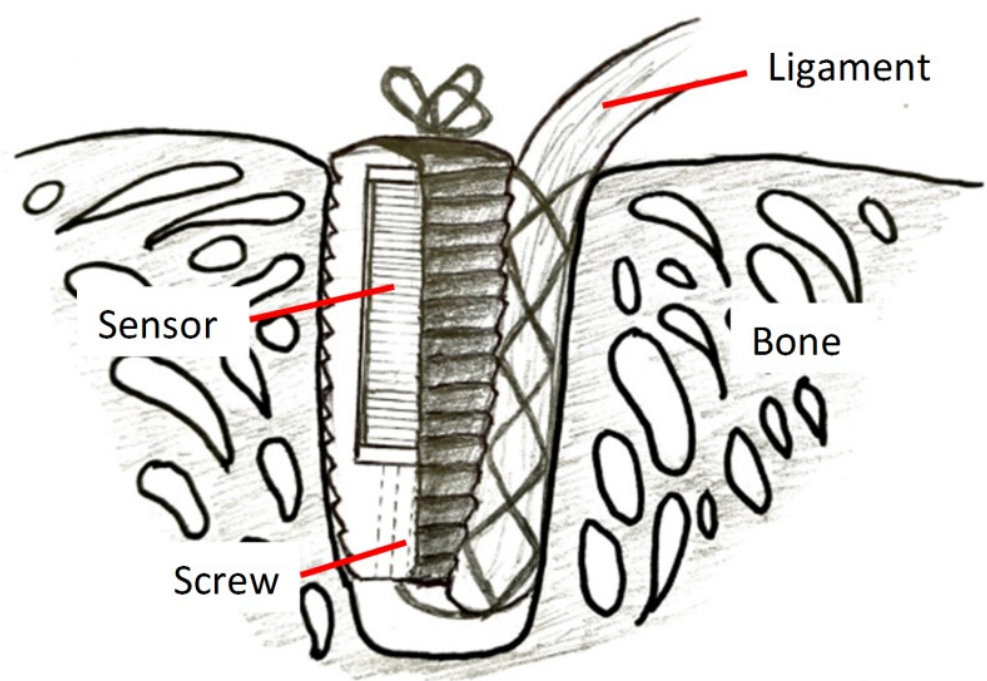

Figure 2.1 Illustration of the designed sensor.

Cross sectional view of the sensorized screw implanted is shown, the sensor inductive winding is represented screws broken view.

The sensorized bone screw was consisted of 2 parts:

i. Bone screw thread: It is the outermost shell of the bone screw and it consists of a threaded exterior and a hexagonal hollow interior. The exterior threading is step tapered to allow for maximum insertion torque when fixing the bone screw. The hexagonal cutout is in two stages with one much wider than the other. The wider portion is towards the top of the screw and extends down till the cutout does not interfere with the tapering thread. The hexagonal cutout drops to a smaller dimension below the larger one.

ii. Temperature sensor: This part houses the LC temperature sensor circuit comprised of the inductive winding, capacitor and the thermistor. The inductive winding is made on a cylindrical shaft with an internal hexagonal cutout. The temperature sensor has a hexagonal top and base which 
corresponds to the hexagonal cutout in the bone screw thread. This allows the sensor portion to be placed inside the screw portion securely. The smaller hexagon allows the screw to be driven with a corresponding hexagonal screw driver.

The hexagonal cutout in the sensor aligns with the smaller hexagonal cutout in the screw thread. This allows the screw to be attached to the head of screw driver which has the corresponding hexagonal head. Usually, the screw drivers are custom designed for both open and laparoscopic surgeries. Screw drivers also have assistive accessories to maintain high precision. The bone screw thread and temperature sensor parts can be put together and fixed using a biocompatible adhesive (Loctite Hysol ${ }^{\circledR}$ medical adhesives or Masterbond EP42HT-2Med epoxy) which confer to ISO 10993 biocompatibility standards. The current model used for test was not biocompatible as the model was a 3D printed prototype.

The screw was designed using SOLIDWORKS ${ }^{\circledR}$, a 3D CAD design software. Different dimensions of the screw were designed and 3D-printed using Lulzbot ${ }^{\circledR}$ Mini for initial prototyping and study. The printing material used was black PLA filament. This initial prototype is not actually meant for implant in an animal or human body. The print material used is not biocompatible. In order to produce a biocompatible model, biocompatible 3D filament should be used or other prototyping techniques such as mold casting should be used. 


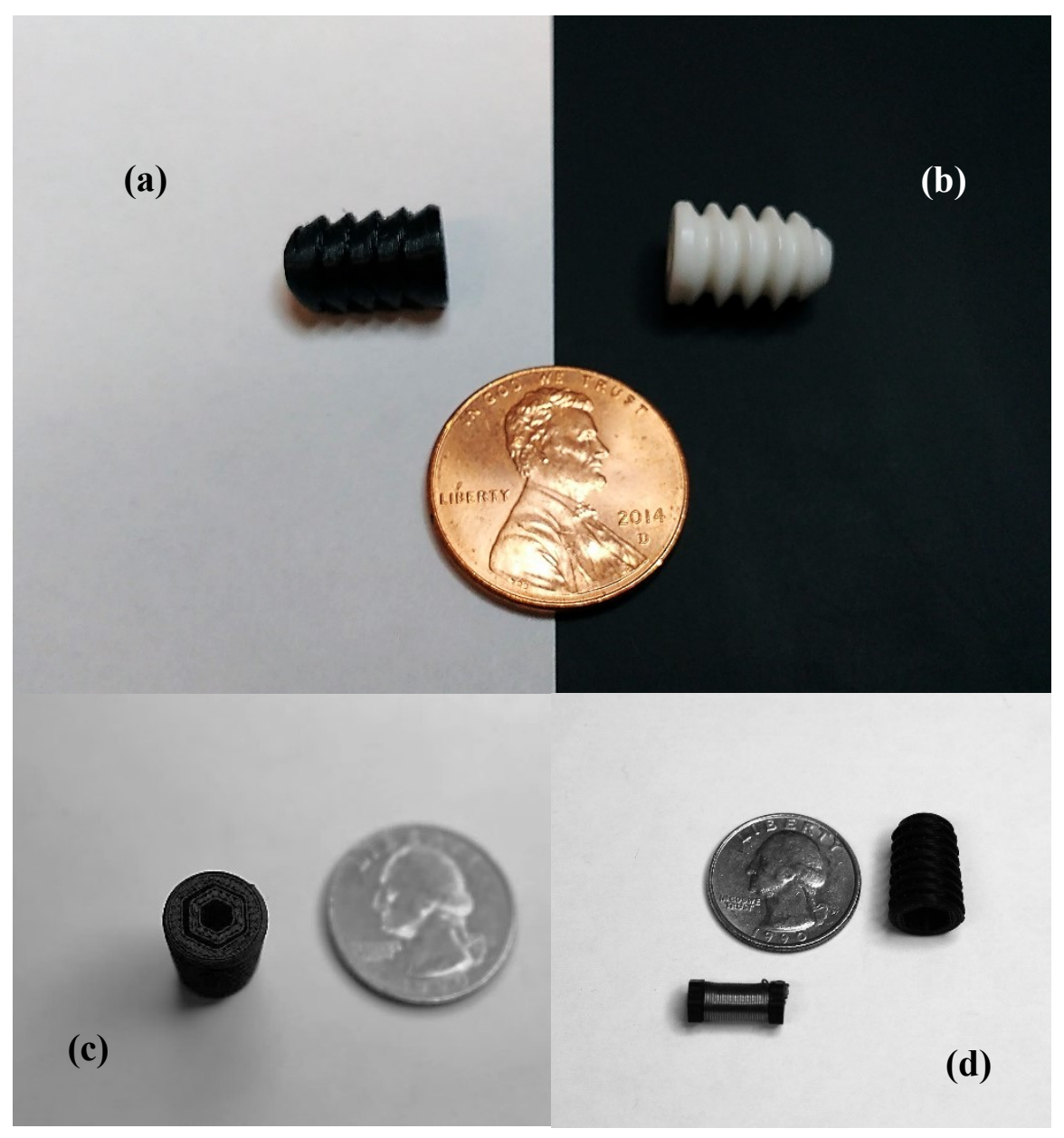

(e)

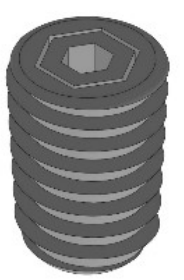

$\leqslant$

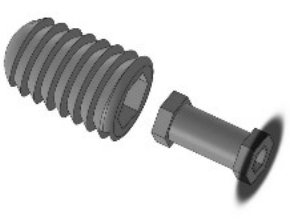

(f)

Figure 2.2 Interference screws.

(a) Designed interference screw $12 \mathrm{~mm} \times 8 \mathrm{~mm}$, (b) referred model of Arthrex Tendonosis biocomposite screw $12 \mathrm{~mm} \times 8 \mathrm{~mm}$ (c) designed interference $18 \mathrm{~mm} \times 12 \mathrm{~mm}$ and (d) bone screw thread and temperature sensor part with the copper winding coil. CAD models of the design (e, f). The hexagonal cut outs can be observed in the figures (c, e and f) 
Table 2.1 Different interference screw sizes designed and their corresponding sensors (max. length $\times$ max. diameter).

\begin{tabular}{|c|c|}
\hline $\begin{array}{c}\text { Screw dimensions } \\
\text { (max. height } \times \text { max. diameter) }\end{array}$ & $\begin{array}{c}\text { Sensor dimensions } \\
\text { (max. height } \times \text { max. diameter) }\end{array}$ \\
\hline $28 \mathrm{~mm} \times 12 \mathrm{~mm}$ & $18 \mathrm{~mm} \times 5 \mathrm{~mm}$ \\
\hline $18 \mathrm{~mm} \times 12 \mathrm{~mm}$ & $14 \mathrm{~mm} \times 5 \mathrm{~mm}$ \\
\hline $12 \mathrm{~mm} \times 8 \mathrm{~mm}$ & $8 \mathrm{~mm} \times 3 \mathrm{~mm}$ \\
\hline
\end{tabular}

The length and diameter of the sensor shaft depends on the overall length and diameter of the screw. Designs can be varied to adjust for different pitches and sizes. The current interference screw model can be used for ligament reconstructions in knee ligaments such as ACL, PCL, LCL and MCL.

\subsection{LC Temperature Sensor}

Several circuit designs were made for the LC circuit with different values of inductance and capacitance. The resonant frequencies of these designs vary between 15 $\mathrm{MHz}$ to $30 \mathrm{MHz}$. The circuit consists of an inductor, a capacitor, and a thermistor in series as shown in Figure 2.2. The thermistor here acts as a transducer. It causes the total resistance in the circuit to vary as the thermistor resistance $R_{t}$ depending on the temperature. 


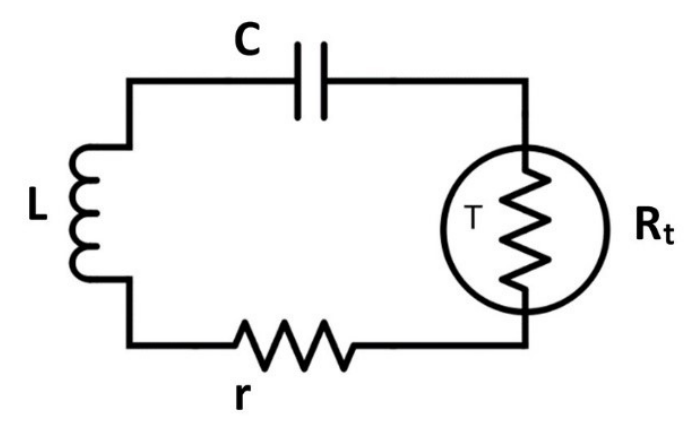

Figure 2.3 Typical serial LCR circuit.

$R_{t}$ represents the thermistor resistance and $r$ represents the circuit resistance this includes the resistance of the copper coil and any resistance during the soldering of the circuit.

The circuit in Figure 2.2 represents a Series Resonant Circuit, with inductance $L$, capacitance $C$, thermistor resistance $R_{t}$ and circuit resistance $r$. The impedance of the circuit $Z_{S}$ is given by the equation

$$
Z_{S}(j \omega)=r+R_{t}+j\left(X_{L}-X_{C}\right)
$$

where inductive reactance $X_{L}$ is expressed as

$$
X_{L}=2 \pi f_{0} L
$$

and the capacitive reactance $X_{C}$ is

$$
X_{C}=\frac{1}{2 \pi f_{0} C}
$$


At the resonant frequency, $Z_{S}$ is minimum in Equation (2.1). As the capacitive and inductive reactance cancel each other out, $X_{C}=X_{L}$ (Equation (2.2) and Equation (2.3)). The resonant frequency can be computed by

$$
f_{0}=\frac{1}{2 \pi \sqrt{L C}}
$$

The half power frequency bandwidth $B W(-3 \mathrm{~dB})$ is determined by

$$
B W=\frac{r+R_{t}}{2 \pi L}
$$

The quality factor $Q$ (Q factor), defined as the ratio of the energy stored in the system per one cycle to the energy dissipated in the system per one cycle, is also given by the ratio of resonant frequency to the half power bandwidth.

$$
Q=\frac{f_{0}}{B}
$$

From Equations. (2.4), (2.5) and (2.6), at resonance 


$$
Q=\frac{1}{r+R_{t}} \sqrt{\frac{L}{C}}
$$

In a circuit (sensor), $L$ and $C$ are constant and the resistance in the circuit is contributed mainly by the resistance of the inductive wire windings. The circuit resistance $r$ remains small and constant. The Q factor thus only depends on the resistance of the thermistor $R_{t}$. In the sensor, we use a NTC thermistor with a small resistance value. The thermistor value was kept constant at $22 \Omega$ with different capacitor values and inductance. It should also be noted that the temperature change can cause changes in inductance and capacitance, which may cause small shift in resonant frequency and Q factor, but it is negligibly small for the relatively small operating temperature of $30-40{ }^{\circ} \mathrm{C}$. The inductor is the coil made by winding magnet copper wire over the cylindrical shaft of the temperature senor. The inductance value (in $\mathrm{H}$ ) for the windings was calculated using the following equation for solenoid inductance.

$$
L=\frac{r^{2} \times N^{2}}{9 \times r+10 \times l}
$$

Where $r$ is the radius of the winding, $N$ is the number of turns and $l$ is the length of windings (in m). Similarly, when there are multilayer windings,

$$
L=\frac{0.8 \times r^{2} \times N^{2}}{6 \times r+9 \times l+10 \times d}
$$


Where $d$ is the depth of the coil windings in $\mathrm{m}$.

The sensors inductor was first made, followed by calculating the inductance. Commonly available capacitor values, ranged in between $1-10 \mathrm{pF}$, was then connected in series to the inductor. With the limited capacitance values, it was not possible to maintain a single resonant frequency for all the fabricated sensor. However, the resonant frequencies were maintained in the range of $15 \mathrm{MHz}$ and $30 \mathrm{MHz}$. In the experiment, it was found that the resonant frequency does not contribute to $\mathrm{Q}$ factor, which is taken as the measure of temperature. The capacitor and thermistor used are surface mounts of the size 0603 (1.6 $\mathrm{mm} \times 0.8 \mathrm{~mm})$. The capacitor and thermistor was arranged by stacking one up on the other, separated by a thin film insulator and then soldering one end to each other and the other two ends to the inductor (see Figure 2.4).

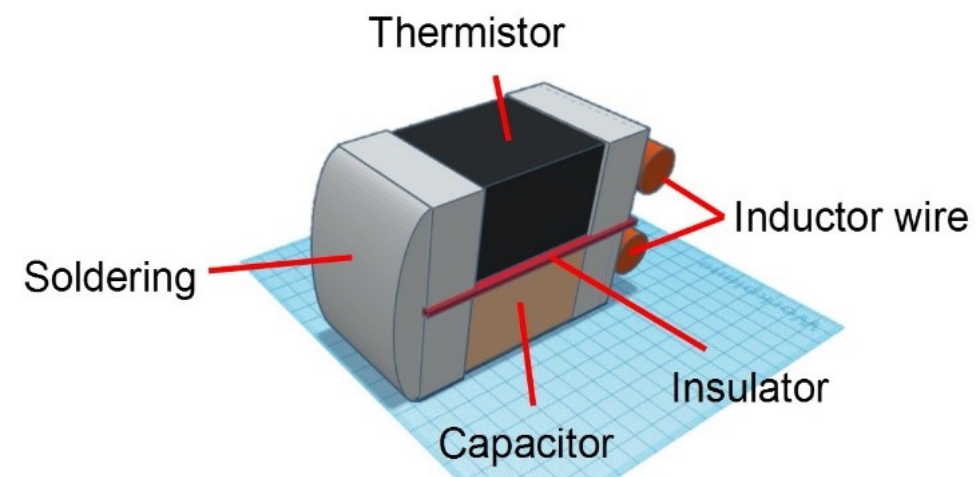

Figure 2.4 Layout of SMD thermistor and Capacitor 


\subsubsection{Thermistor}

Thermistors are resistors which change their resistance value according to the ambient temperature. Negative temperature coefficient (NTC) thermistors were used in the project. In NTC thermistors the resistance decreases as temperature [46]. The Q factor of the sensor is inversely proportional to the resistance in the LC circuit (Equation (2.8)). As the resistance of the NTC thermistor drops with the increase in temperature, the Q factor of the resonance increases. The relationship between temperature and resistance of the thermistor for a narrow range is almost linear.

$$
\Delta R_{t}=k \Delta T
$$

where $k$ is the proportionality constant which is negative in case of NTC thermistor (resistance decreases as temperature increases).

Thermistor usually have a linear relation between temperature and resistance, but this only holds true for a small temperature range. In case of wide temperature range, higher order approximations are required for calibrating the resistance and temperature. In this application, the measurement range is very small $\left(30-40{ }^{\circ} \mathrm{C}\right)$. Therefore, the relation between temperature and Q factor can be considered linear from Equations (2.8) and (2.7).

Self-heating effect of thermistor is another important factor to be considered. The total applied power from the coil is $<10 \mathrm{dBm}$. This power rating is similar to another wireless implantable sensor, CardioMEMS ${ }^{\mathrm{TM}}$ HF System (St. Jude Medical), which also has a transmitting power of $10 \mathrm{dBm}$ or less. Furthermore, the active time of this sensor is 
expected to be relatively short, with at most about 5 minute per 6 hours (assuming collecting a temperature data point 4 times per day). Therefore, we do not expect the wireless interrogation will contribute considerable heating to body tissue to cause damages.

\subsubsection{Detection Coil}

As mentioned earlier, the sensor works on the basis of inductive coupling between the sensor and the detecting coil [53]. The sensor is also interrogated with the help of the same detection coil connected to an impedance analyzer. The impedance analyzer detects the impedance of the sensor using $\mathrm{S}_{11}$ parameter, which is the input reflection coefficient of the scattering parameter. The input reflection is the ratio of applied signal to Port 1 to the reflected signal back on Port 1 of the impedance analyzer [48].

$$
S_{11}=\frac{V_{\text {reflected }}}{V_{\text {applied }}}
$$

This is also equivalent to the reflection coefficient $\Gamma$,

$$
\Gamma=\frac{V_{\text {reflected }}}{V_{\text {applied }}}=\frac{Z_{L}-Z_{S}}{Z_{L}+Z_{S}}
$$


Where $Z_{S}$ and $Z_{L}$ are source and load impedances, respectively. Since the coil and sensor are coupled, the impedance of the coil is also influenced by the sensor. The relation between the impedances of the coil and the sensor was derived by Ong et al., [52] as

$$
Z_{T}=Z_{C}+\frac{\omega^{2} M^{2}}{Z_{S}}
$$

Where $Z_{T}$ is, the total impedance measured across the terminal of the coil, $Z_{C}$ is the coil impedance, $Z_{T}$ is the sensor impedance, and $M$ is the mutual inductance coupling between the sensor and the coil. During a measurement, the impedance of the coil was measured prior to placing the sensor. All sensor measurements were subtracted to the coil impedance to obtain the pure sensor impedance, which is the second term in the right-hand side of Equation (2.13).

The impedance analysis of the LC sensor is carried out by applying a time varying voltage to the detection coil. This produces a magnetic flux as stated by Ampere's Law $[58]$

$$
B_{\emptyset}=\frac{\mu_{0} I}{4 \pi a}\left(\cos \alpha_{1}-\cos \alpha_{2}\right)
$$

Where $I$ is the current, $a$ distance from the center of the wire and $\mu_{0}$ the permeability of free space in the detection coil. This is also consistent with Biot-Savart's Law [58], which 
defines the magnetic field at any point induce by a current carrying conductor. The magnetic field at any point is given by

$$
B_{z}=\frac{\mu_{0} I N a^{2}}{2\left(a^{2}+r^{2}\right)^{3 / 2}}
$$

Where $N$ is the number of turn, $a$ is the radius of the coil, and $r$ is the distance from the center of the coil. The magnetic field determined by this equation falls off with $r^{-3}$ from center of the coil to the point. This distance is the main limiting factor for detection of the sensor since the magnetic field and the inductance coupling decrease with distance.

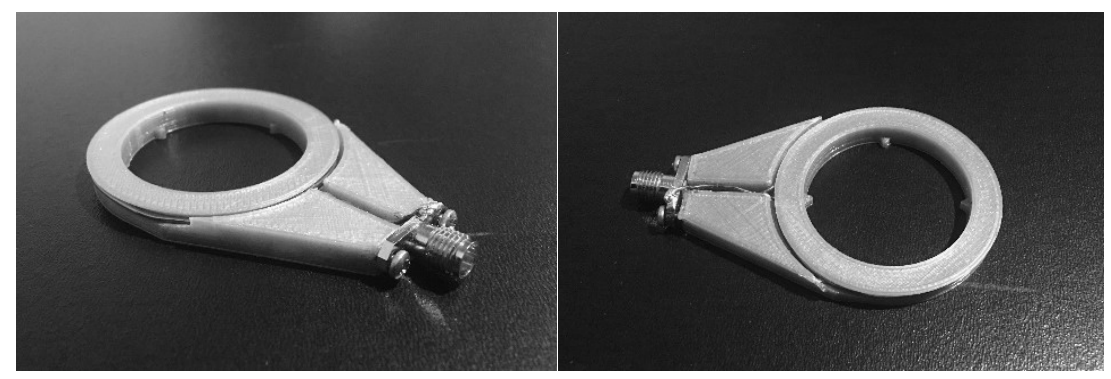

Figure 2.5 Detection coil with $40 \mathrm{~mm}$ radius.

A portion of this field in Eq. (2.15) induces a voltage in the sensor coil, as defined by Faraday's Law of induction [58]. Maximum voltage can be induced by increasing the magnetic field, which can be achieved by varying different parameters such as by increasing $I, N, a$ or reducing $r$. Applied current is determined by the power supplied to detection coil which cannot be increased beyond a specific limit because this causes the 
detection coil to heat up. The number of turns also cannot be increased as more windings cause increase noise susceptibility. The optimum coil diameter was calculated to be around $40 \mathrm{~mm}$ from Eq. (2.15) for a distance of $30 \mathrm{~mm}$, measured from the center of the detection coil to the center of the sensor (Figure 2.6).

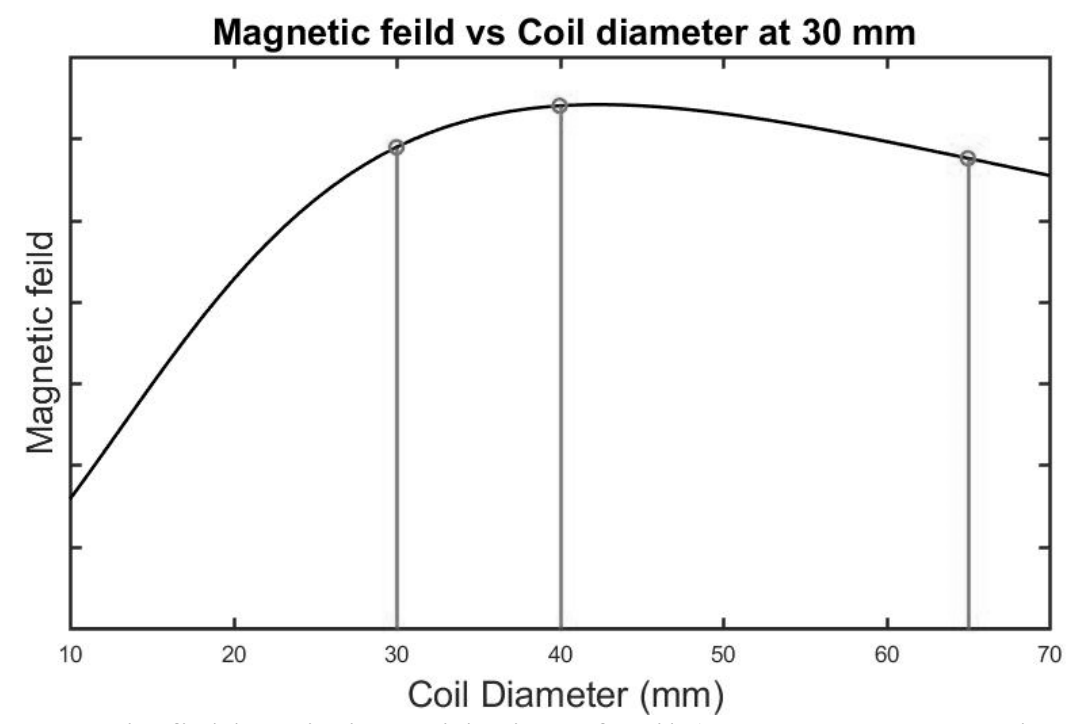

Figure 2.6 Magnetic field variation with size of coil $(30 \mathrm{~mm}, 40 \mathrm{~mm}$ and $65 \mathrm{~mm})$ at 30 $\mathrm{mm}$

The induced voltage in the sensor also gives rise to an opposing magnetic field. The opposing magnetic field causes a voltage drop in the detection coil. The impedance analyzer detects the total impedance of the detection coil, which is the sum of coil impedance and the impedance induced due to the sensor. The sensor can then be characterized by checking the frequency response around the resonant frequency. Sensor characterization is done monitoring two parameters the quality factor and the error $\%$ corresponding to temperature and quality of the signal. 


\subsection{Q factor}

As calculated in Section 2.3 (Equation (2.7)), quality factor is determined from the frequency response. The $\mathrm{Q}$ factor as shown in the figure was calculated from the raw frequency response data using a Matlab code. The Matlab code checks the peak impedance to determine the resonant frequency and then determines the half power point $(-3 \mathrm{~dB})$ to determine the Bandwidth. The Q factor was then calculated from the resonant frequency and bandwidth. Polynomial fitting was used to reduce the effects of noise component in determining the peak and bandwidth. Figure below demonstrates the noise and the calculated Q factor and Error \%.

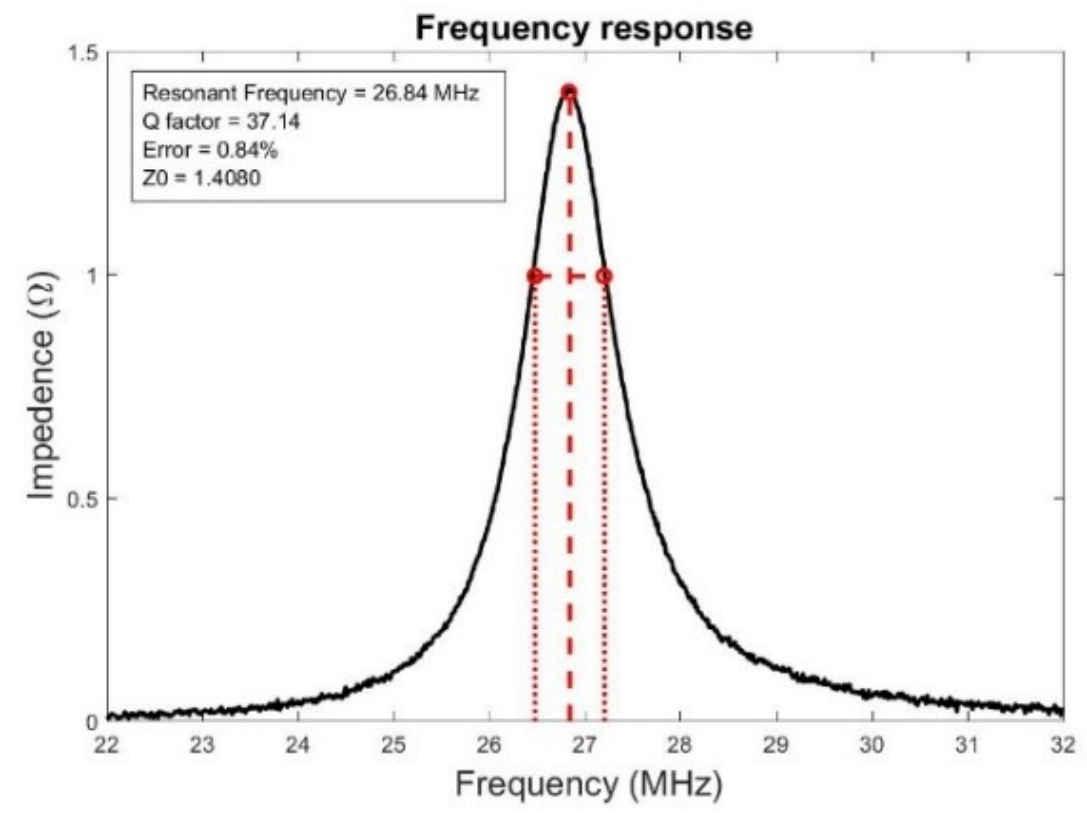

Figure 2.7 Frequency response plot for sensor $0 \mathrm{~mm}$ from of $30 \mathrm{~mm}$ detection coil 


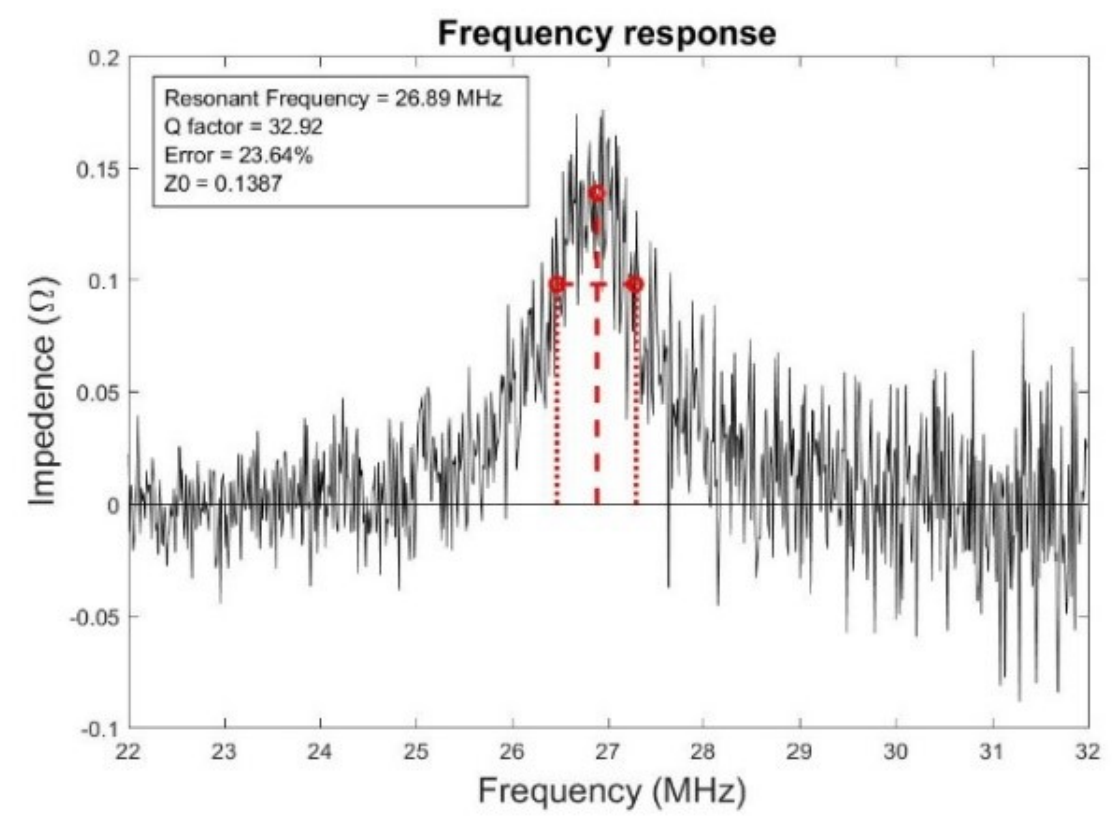

Figure 2.8 Frequency response plot for sensor $20 \mathrm{~mm}$ from of $65 \mathrm{~mm}$ detection coil

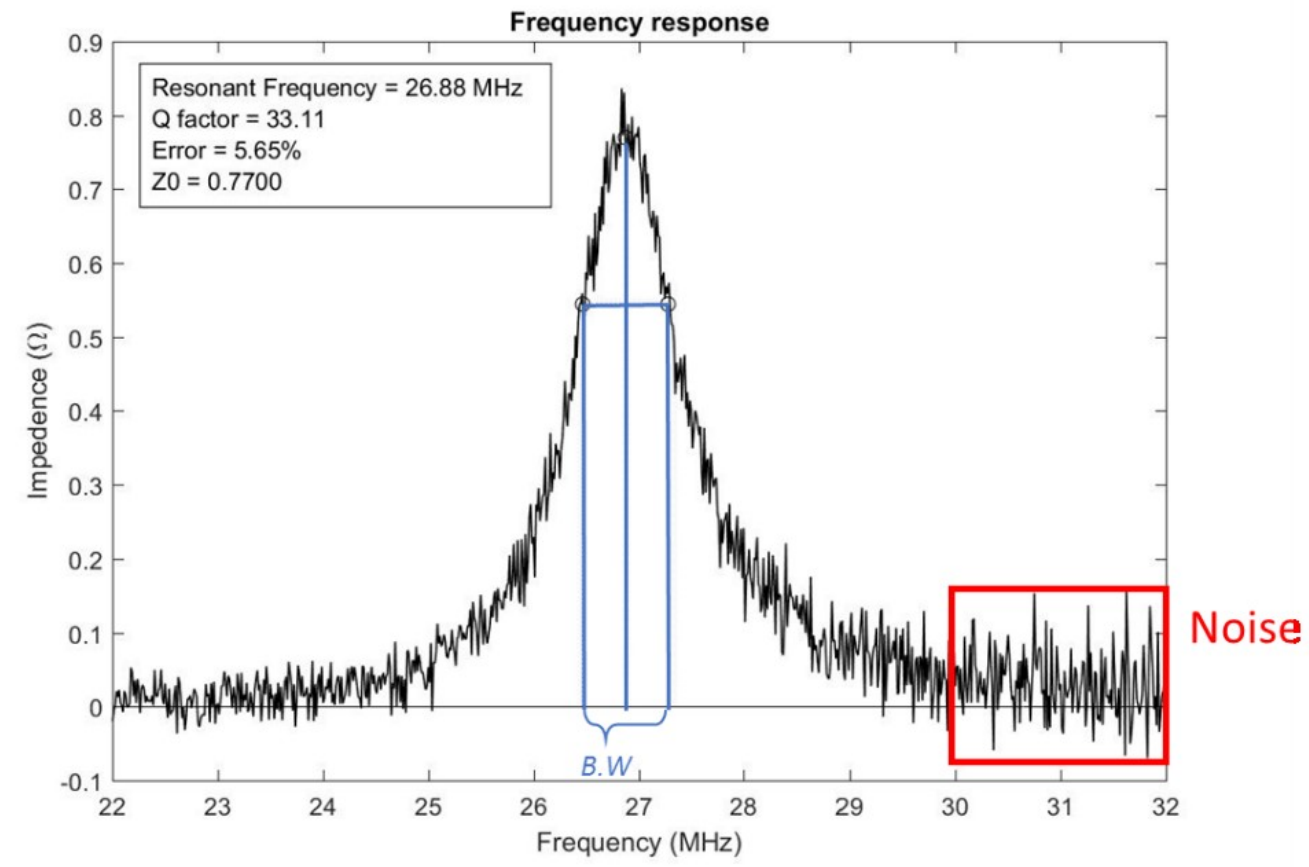

Figure 2.9 Bandwidth, resonant frequency and noise for a typical frequency response. The bandwidth and resonant frequency is used calculate $\mathrm{Q}$ factor (blue) and maximum measured noise from which the Error\% is calculated. 


\subsection{Error \%}

Error $\%$ is used to represent the quantitative measure of noise (Figure 2.9), it is defined here as the percent of standard deviation in the frequency response to the peak response.

$$
\text { Error } \%=\frac{\text { Standerd Deviation ofmax. noise }}{\text { Max.impedence at resonant frequency }} \times 100
$$

It effectively determines the amount of noise and the variability in the calculated resonant frequency and Q factor. It was observed that the best signal response had Error \%

of less than $1 \%$, Q factor could be efficiently determined for up to $30 \%$. But an Error $\%$ of less than $10 \%$ percent is considered efficient for this study. 


\section{Experiment and Results}

Experiments were conducted in 3 phases. Initial test was performed to optimize the detection coil to determine the role of coil diameter, distance of separation between the sensor and the detection coil, wire diameter, number of windings of the detection coil and power supplied to the detection coil. Next, the sensor design was varied to determine the best sensor. Upon determining the best coil and sensor, the performance of the sensor was evaluated in terms of sensitivity. Repeatability, and the relative positioning of the sensor from the detection coil.

As described in the previous Section 1.6.1, a network analyzer was used to measure the impedance of the sensor with a detection coil. The Network/Spectrum analyzer, Agilent 4396B, was used to determine the impedance of the sensor by measuring the $\mathrm{S}_{11}$ response of the detection coil. The network analyzer was connected to a PC via GPIB (General Purpose Interface Bus) connection. The network analyzer was remotely controlled using a custom Visual Basic program, which recorded the frequency response in $\mathrm{S}_{11}$ mode for a specified frequency range, depending on the resonant frequency of the sensor used. The sensor impedance was determined by subtracting the measured frequency response of the coil with the sensor to the measurement of the coil's impedance without the sensor. The raw data was further analyzed in Matlab to obtain the Q factor and Error \%.

The data was averaged in the impedance analyzer at a factor of 150 . And the data was collected in a $10 \mathrm{MHz}$ window around the resonance frequency of the sensor with 400 data points. 


\subsection{Detection Coil Optimization}

\subsubsection{Distance between Sensor and Detection Coil}

The range of detection is a major concern for this study. The distance was selected as $30 \mathrm{~mm}$, which is a conservative measure of interference screws placement in Medial Collateral Ligament (MCL) reconstruction surgery. A similar distance is observed for Lateral Collateral Ligament (LCL) reconstructions as well. In many cases the same distance can be used for Anterior Cruciate Ligament (ACL) reconstructions. In the case of Posterior Cruciate Ligament (PCL) reconstruction, the detection distance may vary due to muscle and fat tissues of thigh and leg. The inductive coupling reduces with distance according to Equation (2.15).

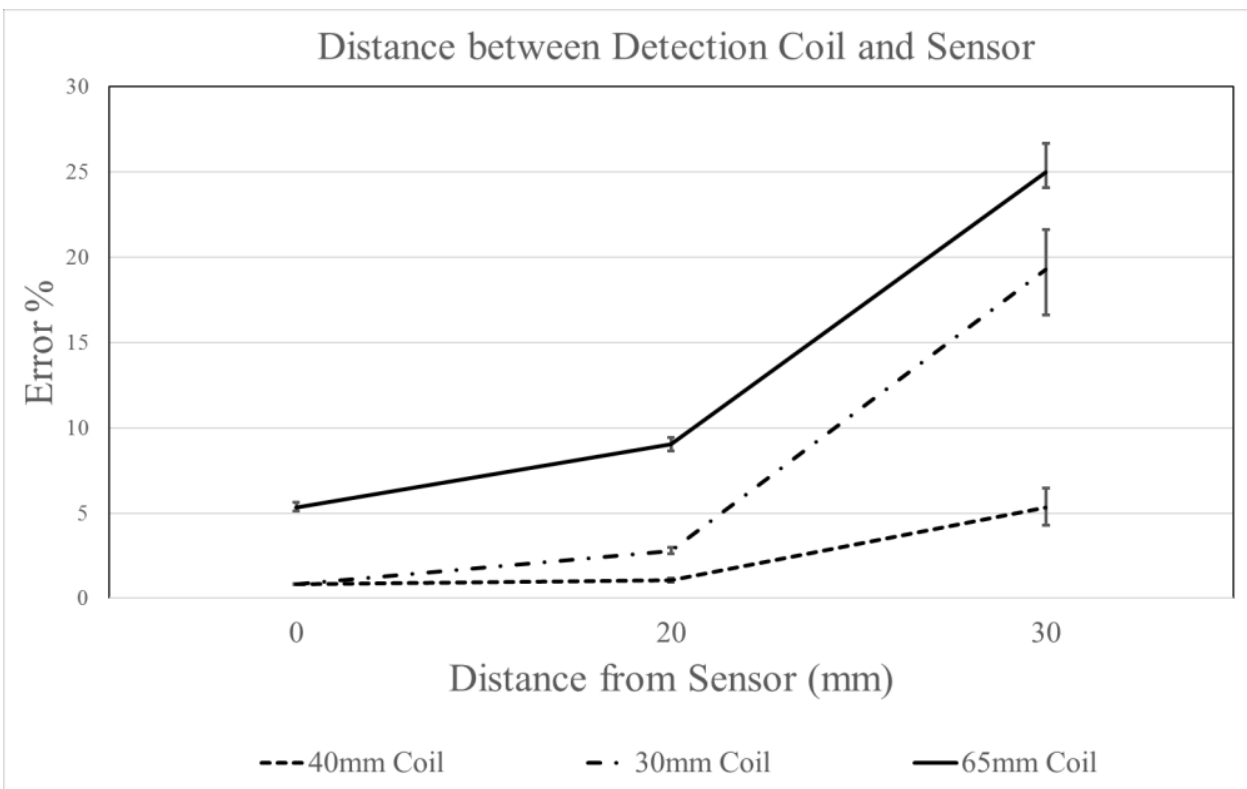

Figure 3.1 Sensor response for change in distance of separation of sensor and coil and diameter of coil.

$40 \mathrm{~mm}$ diameter has the lowest Error \% than the rest. 
Figure 3.1 shows that the $40 \mathrm{~mm}$ coil produce the best response when monitored from a distance of $30 \mathrm{~mm}$. This observation is consistent with the theory described in Equation (2.15). For an increase in the separation distance between the sensor and detection coil, the coil diameter should be increased to maintain a maximum coupling (thus maximum signal). Figure 3.2 shows the response of a sensor when monitored by coils of different diameters at $0 \mathrm{~mm}$ separation. It can be observed that the $30 \mathrm{~mm}$ coil is only marginally better than the $40 \mathrm{~mm}$ coil, which shows that smaller coils are better for sensors implanted near the skin. Nevertheless, $40 \mathrm{~mm}$ coil can be used efficiently from $0 \mathrm{~mm}$ to 30 mm distance.

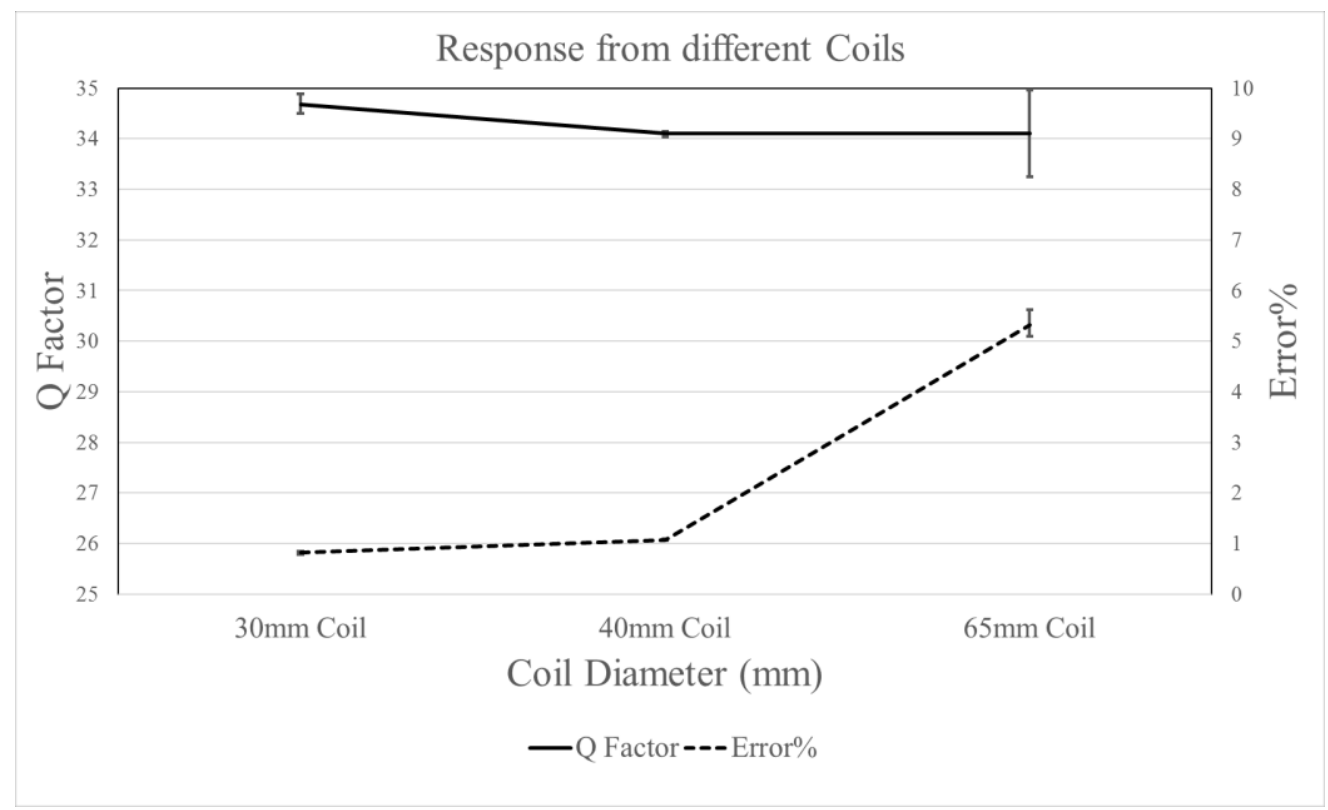

Figure 3.2 Sensor response for change in coil diameter at $0 \mathrm{~mm}$ separation.

Larger coils can be used for deeper sensor placement measurements, but to maintain the good sensor response the power must be compensated enough. Figure 3.2 also shows that the Q factor remains relatively constant throughout the test and the distinguishing 
factor of performance is Error \%. During this test power was kept constant at $5 \mathrm{dBm}$ and all the coils had 2 turns with a 24 AWG wire size.

\subsubsection{Coil Wire Diameter}

The experiment was carried out to determine how the diameter of the wire effects the sensor response. In this experiment, the number of turns was kept constant as 2 and the power was at $5 \mathrm{dBm}$ and the sensor was placed at $20 \mathrm{~mm}$ away from the detection coil. Three different gauges of wire were used in the experiment: 28 AWG, 24 AWG and 18 AWG. The results from Figure 3.3 shows a considerable increase in performance in terms of Error \%, but it is only observed as we go from 28 AWG to 24 AWG. The same is not observed from 24 AWG to 18 AWG wire.

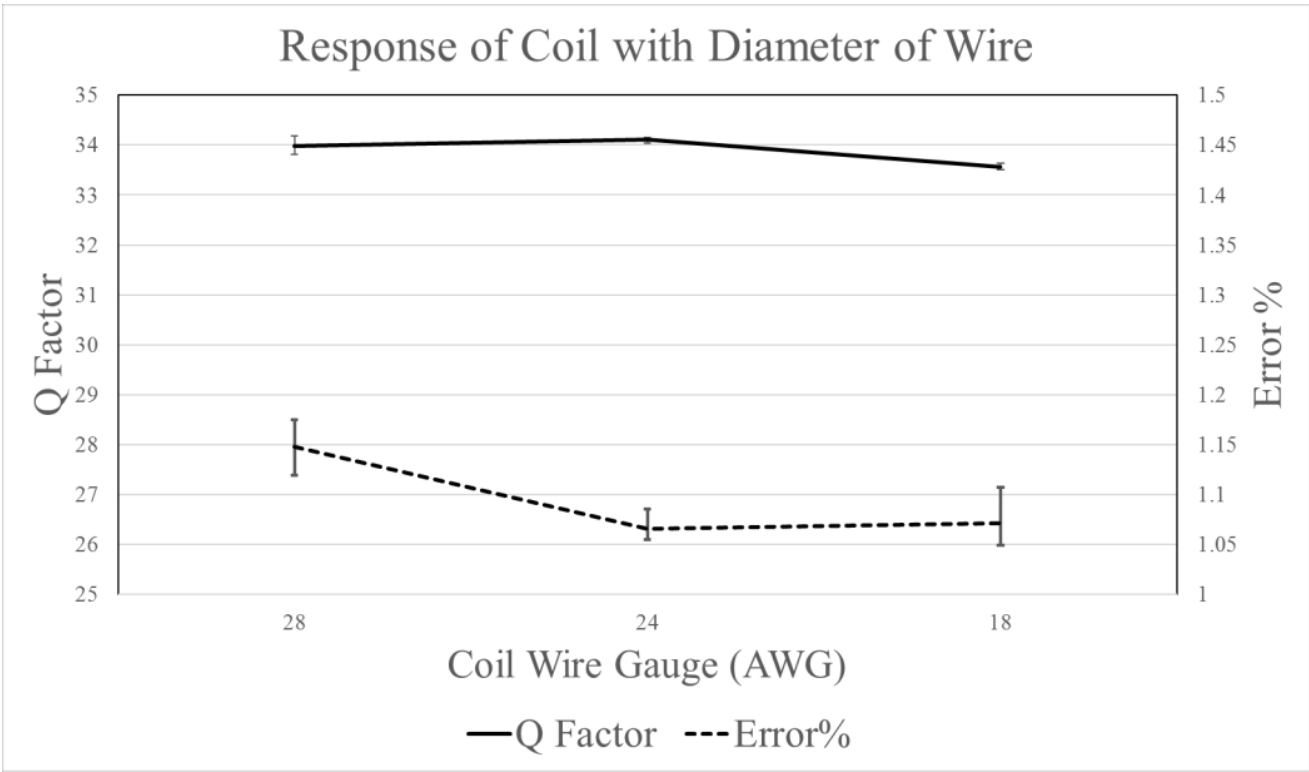

Figure 3.3 Sensor response for different diameters of the wire. 
The observations in Figure 3.3 can be explained by considering the resistance of the magnet wire. The amount of current flowing through the wire depends on the resistance of the wire as the impedance analyzer produces a voltage signal across the detection coil. The resistance of different wire gauges is given in Table 3.1 Wire gauge and resistance.. The resistance difference between 18 AWG and 24 AWG is $\sim 0.06 \Omega$ per meter. Similarly, the difference in resistance between $18 \mathrm{AWG}$ and $16 \mathrm{AWG}$ is $\sim 0.004 \Omega$ per meter. Since the resistance change between $18-16$ AWG is an order of magnitude smaller than the change between $18-24$ AWG, there was no significant performance improvement for 16 AWG wire over 18 AWG wire.

Table 3.1 Wire gauge and resistance.

\begin{tabular}{|c|c|c|}
\hline Wire size (AWG) & Dia. in (mm) & $\begin{array}{c}\text { Resistance per 1000 } \\
\text { ft. }\end{array}$ \\
\hline 18 & 1.024 & 6.39 \\
\hline 20 & 0.813 & 10.1 \\
\hline 22 & 0.643 & 16.2 \\
\hline 24 & 0.511 & 25.7 \\
\hline 26 & 0.404 & 41.0 \\
\hline 28 & 0.320 & 65.3 \\
\hline
\end{tabular}

\subsubsection{Number of Windings in the Coil}

According to Equation (2.15), the magnetic field produced by the coil should increases with the increasing number of windings in the coil. During initial bench tests with 
4 or more turn coils, it was observed that the quality of signal was very low (high Error \%) and it was difficult to calculate the $\mathrm{Q}$ factor from the data. The same was also observed in the single turn wound coil, which was not able to induce enough voltage at $20 \mathrm{~mm}$ height to produce a response.

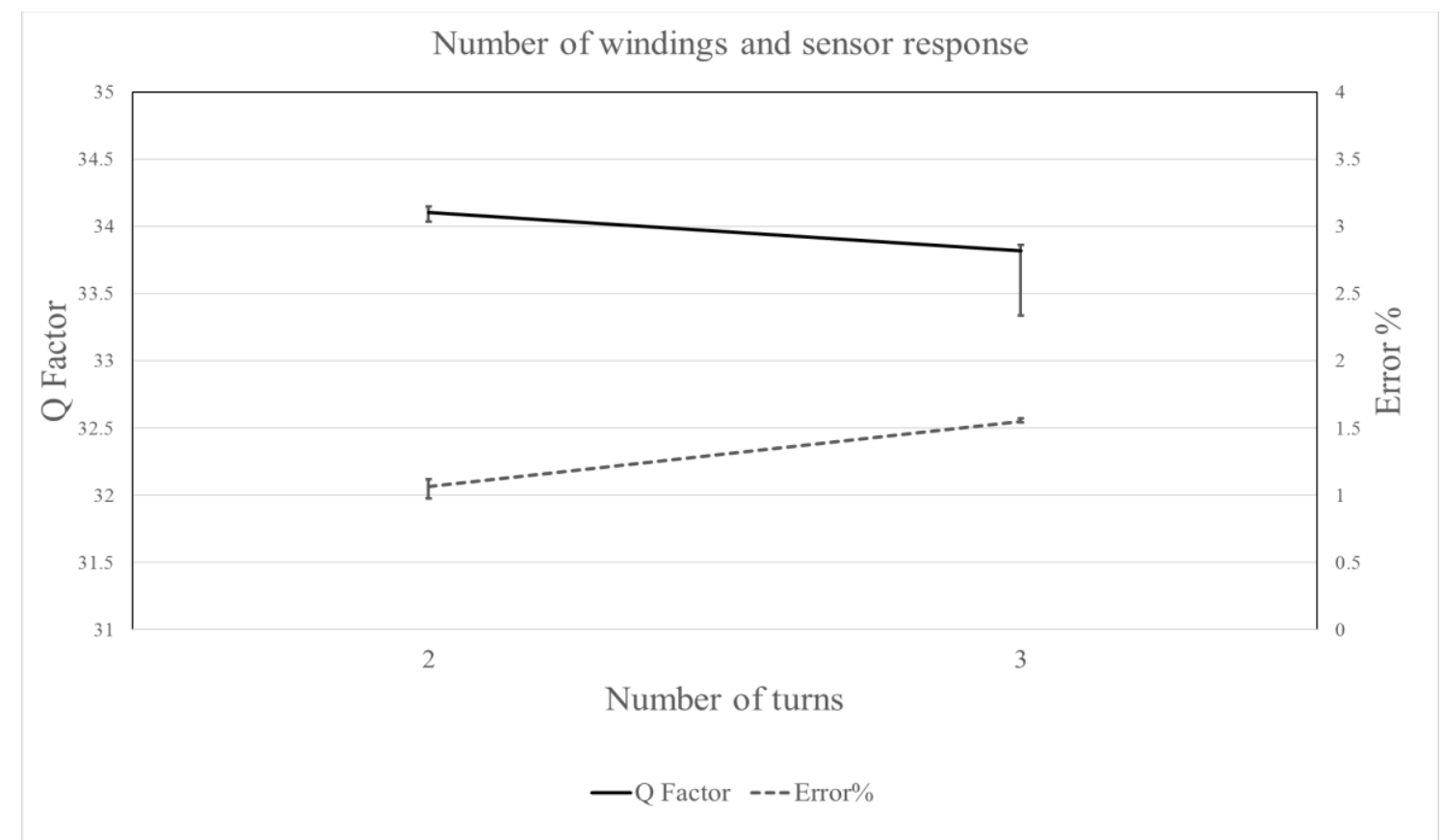

Figure 3.4 Sensor response with different number of windings. Error \% is higher in 3 -turn coil than in 2 turn coil.

This test was conducted using 2 and 3 turn detection coils. The separation distance of the coil was kept constant at $20 \mathrm{~mm}$ and measurement was taken with $40 \mathrm{~mm}$ diameter coil of 24 AWG. Figure 3.4 demonstrates a clear increase in Error \%. This Error \% increase can be attributed to ambient noise picked up by the coil with more number of turns. 


\subsubsection{Power}

According to Equation (2.15), the increase in current causes the magnetic field to increase. In the network analyzer, the power of the output signal can be increased to produces higher current in the detection coil. This will produce higher magnetic field and better sensor performance Figure 3.5. The increased current can also cause power to be dissipated as heat by heating up the detection coil. The power should be maintained low enough to reduce this heating effect. In the experiment the maximum power applied was $10 \mathrm{dBm}$. The unit of power used here is $\mathrm{dBm}$ (decibel-milliwatt). It is defined as the measure of power ratio of signal to one milliwatt in decibel units. It is the typical unit of power in the case of radio frequency and microwave signals.

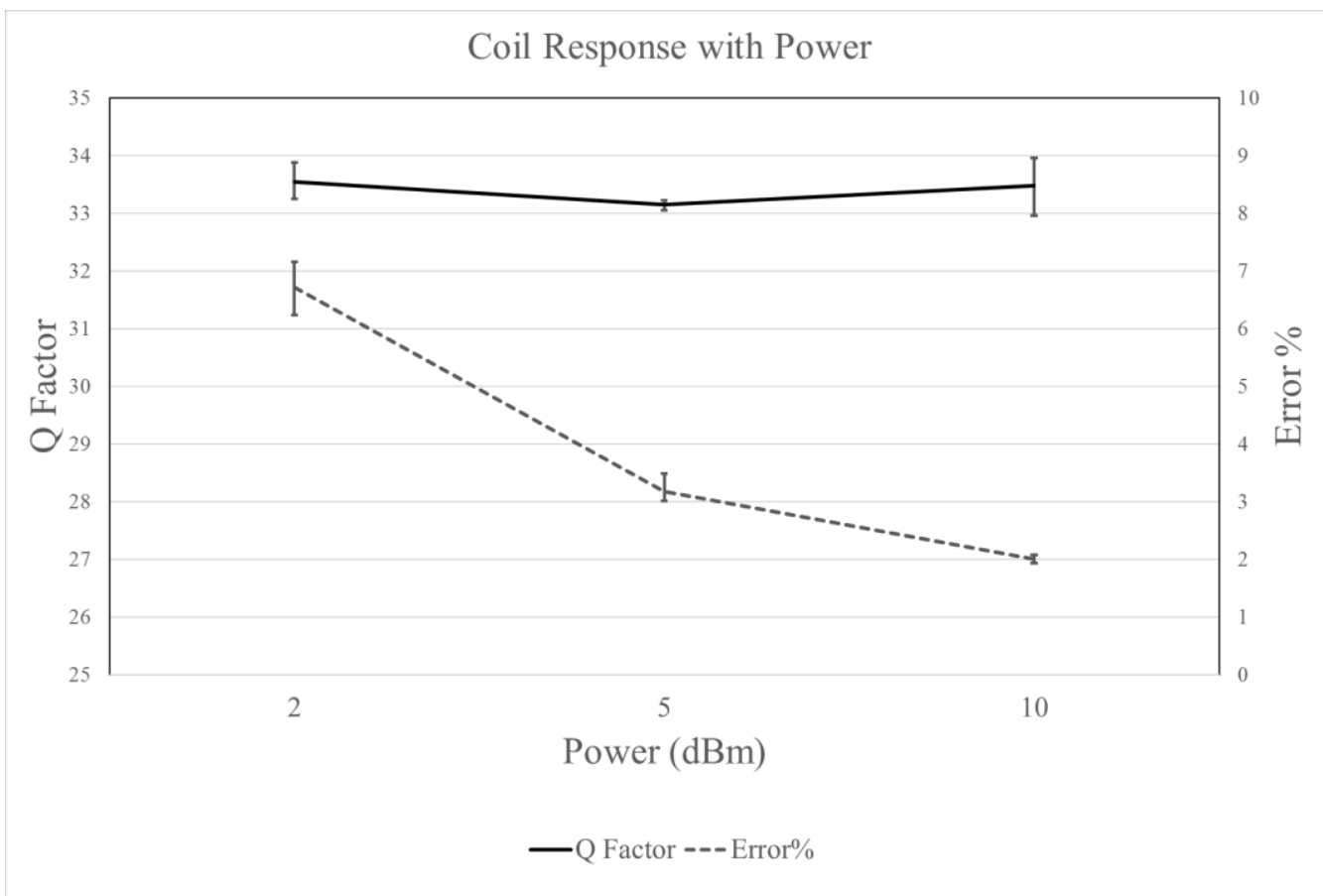

Figure 3.5 Sensor response with change in power applied to the detection coil. Sensor response increases with applied power. 
In this experiment, 3 different values of power were applied $2 \mathrm{dBm}, 5 \mathrm{dBm}$ and 10 $\mathrm{dBm}$. The distance of separation was kept constant at $20 \mathrm{~mm}$. A 2-turn coil of diameter 40 $\mathrm{mm}$ and wire of gauge $18 \mathrm{AWG}$ was used.

\subsubsection{Result}

The detection coil performance was characterized based on the coil diameter, separation distance, number of coil windings, diameter of the wires used and power applied to the coil. The optimum diameter of the coil is dependent on the equation of magnetic field Equation (2.15). When the distance of separation of the coil and sensor was $30 \mathrm{~mm}$, the calculated optimum coil diameter was $40 \mathrm{~mm}$. The experiments verified the performance of the $40 \mathrm{~mm}$ coil as compared to $30 \mathrm{~mm}$ and $65 \mathrm{~mm}$ coils. Equation (2.15) also determines the magnetic field with respect to the number of windings. The magnetic field increases proportionally to the square of the number of turns, however, in the experiment it was determined that the maximum number of turns that could be used was 3 . This was due to the increase in noise in the acquired data for coils with 4 or more windings. It was also noted that the 2-turn coil had lesser Error \% than 3-turn coil, so 2 turns are considered optimum for the detection coil. The diameter of the wire determines the resistance of the wire. As the diameter of the wire increases, the resistance decreases. Therefore, higher diameter wires are better suited for the detection coil, however, in experiments the performance did not increase proportionally with decrease in wire gauge (increase in diameter). This is due to the fact that the resistance change in lower wire gauge is constant. For example, the resistance difference between $18 \mathrm{AWG}$ and $24 \mathrm{AWG}$ is $\sim 0.06$ 
$\Omega$ per meter. Similarly, the difference in resistance between 18 AWG and 16 AWG is $\sim 0.004 \Omega$ per meter. Hence, 18 AWG is considered optimum wire gauge for coil design. In the final test, an increase in power allowed better coupling by producing more current in the coil. The optimized design of the detection coil for a distance of separation of $30 \mathrm{~mm}$ is a $40 \mathrm{~mm}$ diameter coil with 2 turns of $18 \mathrm{AWG}$ magnet wire used at a power rating of $10 \mathrm{dBm}$.

\subsection{Sensor Optimization}

\subsubsection{Different Sensors}

The main parameter of focus in the sensor optimization study was the inductive winding of the sensor. The inductance can be calculated form Equations (2.7) and (2.8). An increase in number of turns in the inductor produces better inductive coupling between the sensor and detection coil. Considering Equation (2.7), the Q factor varies with the inductance $L$ and capacitance $C$. Q factor is proportional to the square root of the ratio of inductance Land capacitance $C$. The thermistor used in all the designs was $22 \Omega$. Similar to the detection coil optimization, different windings were studied for same temperature sensor model $(14 \mathrm{~mm} \times 5 \mathrm{~mm})$. The gauge of the wire and number of turns varied the response of the coil. In the experiment, two sensors with different number of turns and wire diameters were compared to a reference design. As shown in Table 3.2, Sensor 'a' was

considered as the reference sensor, Sensor 'c' had double the number of turns as compared to Sensor ' $a$ ' and Sensor ' $b$ ' had more than twice the wire diameter. 
Table 3.2 Different sensor designs tested

\begin{tabular}{|c|c|c|c|c|c|}
\hline Sensor & $\begin{array}{c}\text { Number of } \\
\text { turns }\end{array}$ & $\begin{array}{c}\mathbf{L} / \mathbf{C} \text { ratio } \\
(\mathbf{H} / \mathbf{F})\end{array}$ & $\begin{array}{c}\text { Wire } \\
\text { diameter } \\
(\mathbf{A W G})\end{array}$ & $\begin{array}{c}\text { Resistance } \\
\text { of the coil } \\
(\mathbf{\Omega})\end{array}$ & $\begin{array}{c}\mathbf{Q} \text { factor (at room } \\
\text { temperature 23 }\end{array}$ \\
\hline $\mathrm{a}$ & 38 & $0.356 \times 10^{6}$ & 36 & 1.628 & 33.649 \\
\hline $\mathrm{b}$ & 38 & $1.1 \times 10^{6}$ & 28 & 0.254 & 45.425 \\
\hline $\mathrm{c}$ & 80 & $7.53 \times 10^{6}$ & 36 & 3.419 & 49.941 \\
\hline
\end{tabular}

The experimental results show that both increase in wire diameter and number of windings increase the $\mathrm{Q}$ factor. This demonstrated by the higher $\mathrm{Q}$ factors produced by Senor ' $b$ ' and ' $c$ ' when compared to Sensor ' $a$ ' while the Error \% remained relatively stable. The limiting factor in the sensor performance is the size of the screw which determines the dimensions of the inductance of the coil, a wider sensor can produce a higher inductance and inductive coupling according to Equation (2.7). The capacitor values can be varied to control the resonant frequency of the sensor. 


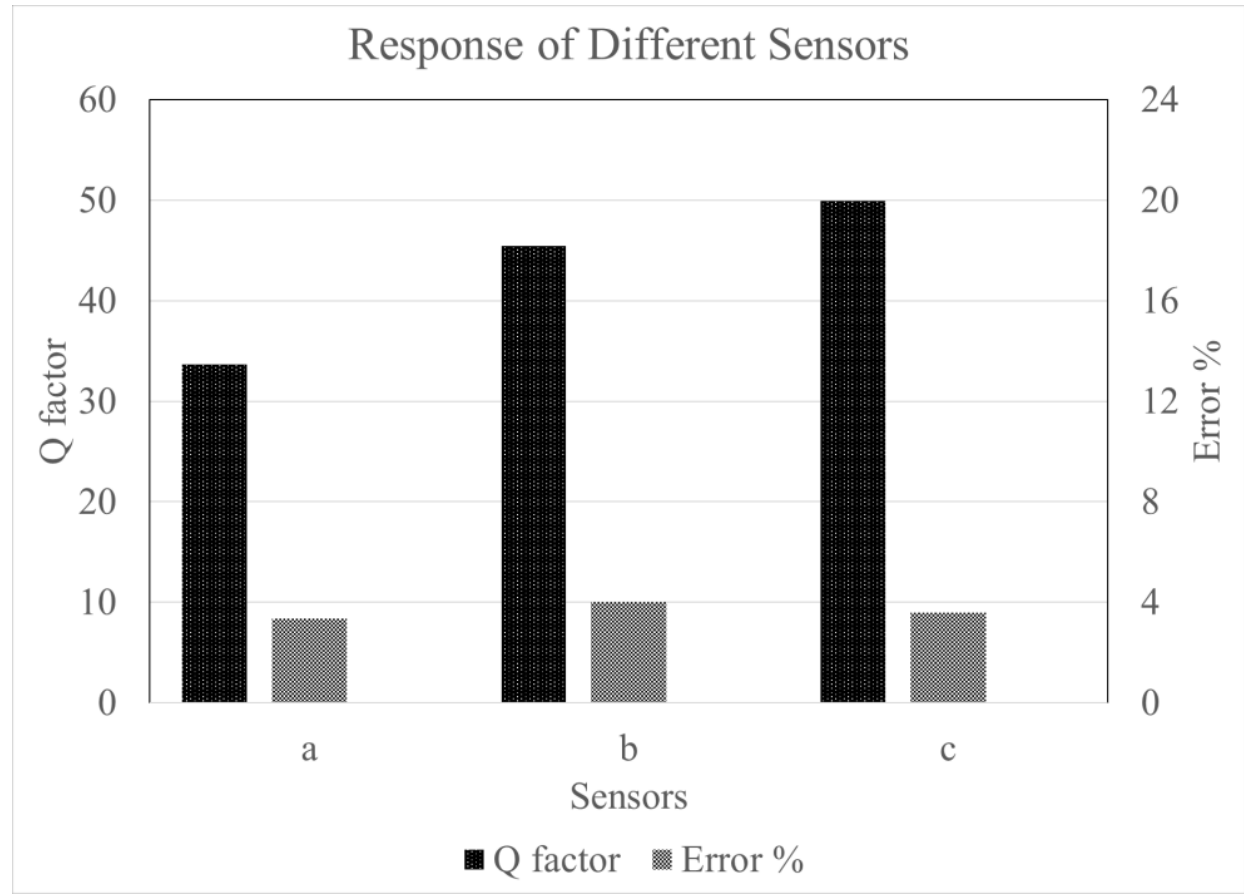

Figure 3.6 Response of different sensor designs.

\subsection{Relative Position Sensors and Detection Coil}

The relative position of the sensor and the angle was measured to evaluate how these parameters impact the sensor performance. In case of an implanted sensor, the relative position and angle cannot be determined after implantation. There needs to be a high window of tolerance for the relative positioning of the sensor response.

Figure 3.7 and Figure 3.8 demonstrate how the relative positioning of the sensor impacts the sensor response. In the case of relative position of the sensor with the detection coil, the sensor response remained the same in terms of Error \% and Q factor when the sensor was within the detection coil's inner loop. The response dropped considerably at 20 $\mathrm{mm}$ form the center of the coil, which was right above the $40 \mathrm{~mm}$ diameter detection coil and no response was detected beyond the $20 \mathrm{~mm}$ distance. 


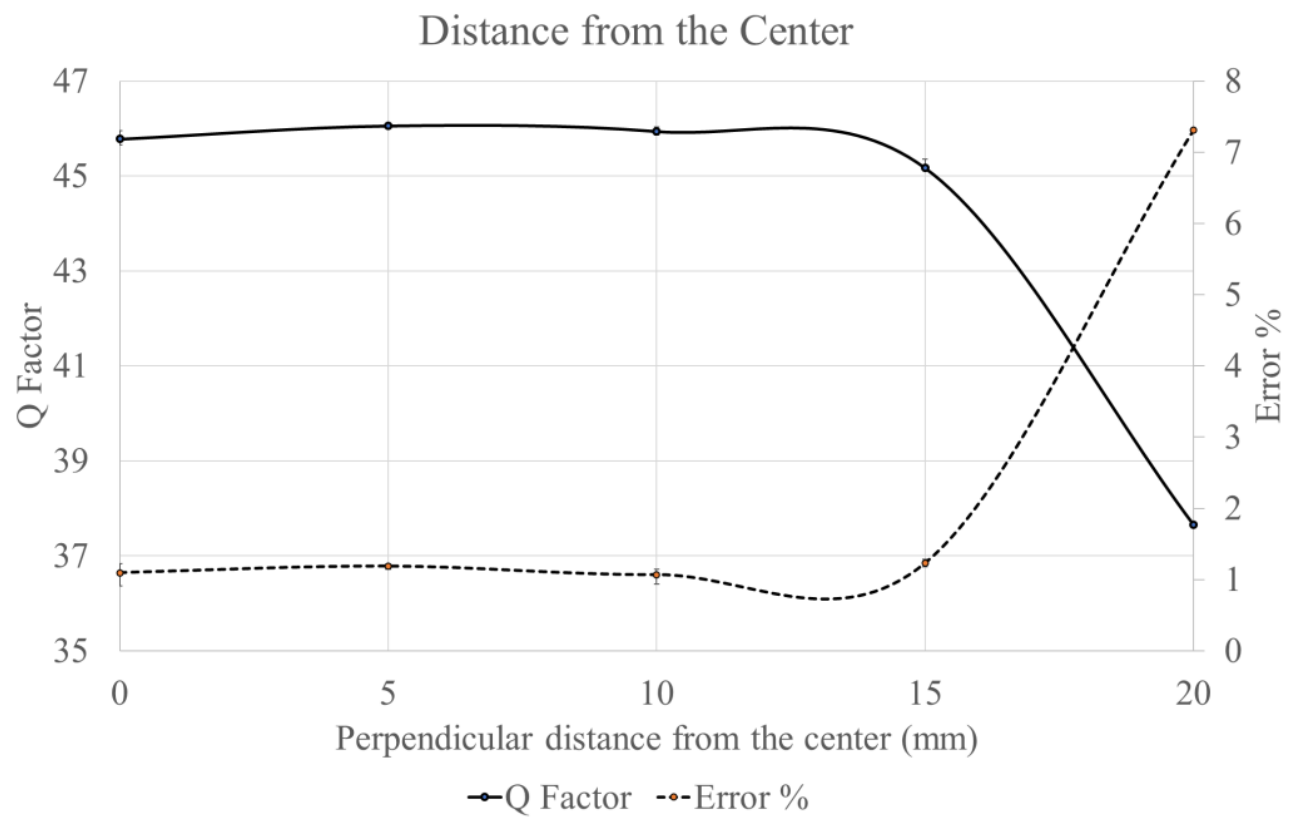

Figure 3.7 Sensor response with change in perpendicular distance between the cylindrical axes of the sensor and detection coil.

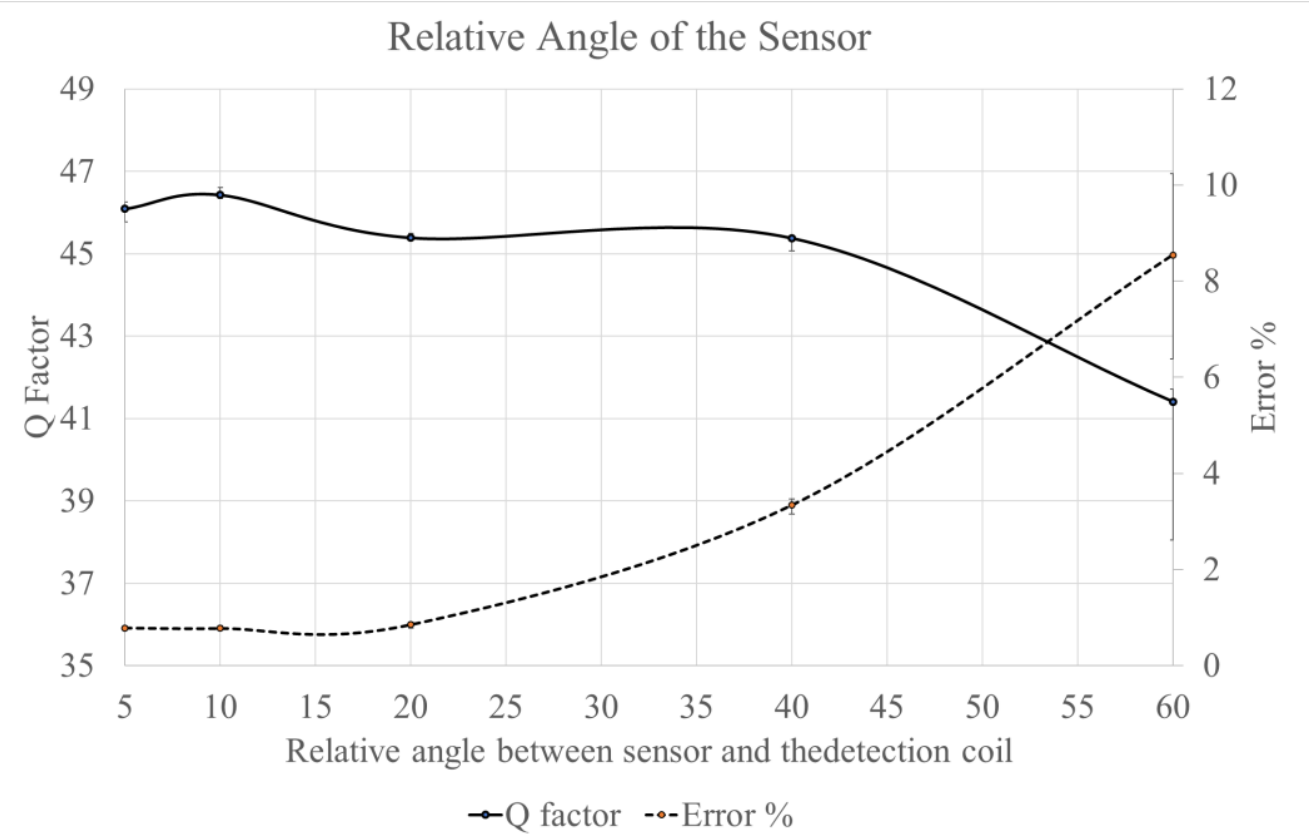

Figure 3.8 Sensor response with change in angle between cylindrical axes of the sensor and detection coil. 
In the case of relative angle measured between the axes perpendicular to the plane of the detection coil windings and the sensor coil windings, there is relatively higher Error $\%$ beyond the $40^{\circ}$. The sensor response was detectable till $60^{\circ}$ with Error \% less than 10 . It is assumed that in medial collateral ligament and lateral collateral ligament reconstruction surgery, the angle should not be more than $30^{\circ}$ perpendicular to the medial epicondyle. The angle of insertion of screw with respect to the plain of insertion (lateral condyle) is higher in ACL reconstruction. This angle makes it possible to interrogate the sensor from the same position as in MCL or LCL reconstruction.

\subsection{Sensor Performance}

Sensor performance was characterized by using the optimized detection coil and sensor designs. The sensor response with respect to temperature was measured to determine its sensitivity and stability of the sensor. A LM35 sensor was used to independently measure the temperature. It is a precision integrated-circuit temperature sensor developed by Texas Instruments [59]. Initial bench test had shown that the temperature tests had errors due to excitation delay between the thermistor in the sensor and the LM35. In order to overcome this response delay, all the temperature readings of the sensor were normalized to that of $22 \Omega$ thermistor. The data obtained between the thermistor resistance and the sensor's Q factor is as shown in Figure 3.9. The temperature characteristics as shown in Figure 3.10 was determined by calibrating thermistor resistance to temperature for LM35 in a static temperature test. 
The temperature test was conducted by placing the sensor and the detection coil inside an enclosed chamber. The chamber was supplied with heated air from a heater to increase the temperature. The temperature of the air and the air flow were controlled from the heater. The temperature was then monitored over several cycles of heating and cooling. The temperature range for sensitivity test was between $30^{\circ} \mathrm{C}$ and $40{ }^{\circ} \mathrm{C}$, and the same for repeatability test was $35^{\circ} \mathrm{C}$ and $45^{\circ} \mathrm{C}$. The resistance of the thermistor and the output of the LM 35 was measure using a high precision Keithley 2001 multimeter.

\subsubsection{Sensitivity}

The results in Figure 3.9 show the linearity of thermistor resistance and Q factor with respect to temperature change. The Q factor vs temperature plot (Figure 3.10) was obtained from the calibration of thermistor resistance and the temperature reading from LM35. As mentioned in Section (2.2.1), over short temperature ranges the thermistor response was linear. This result was observed in the $\mathrm{Q}$ factor vs temperature plot and the thermistor resistance calibration data. 


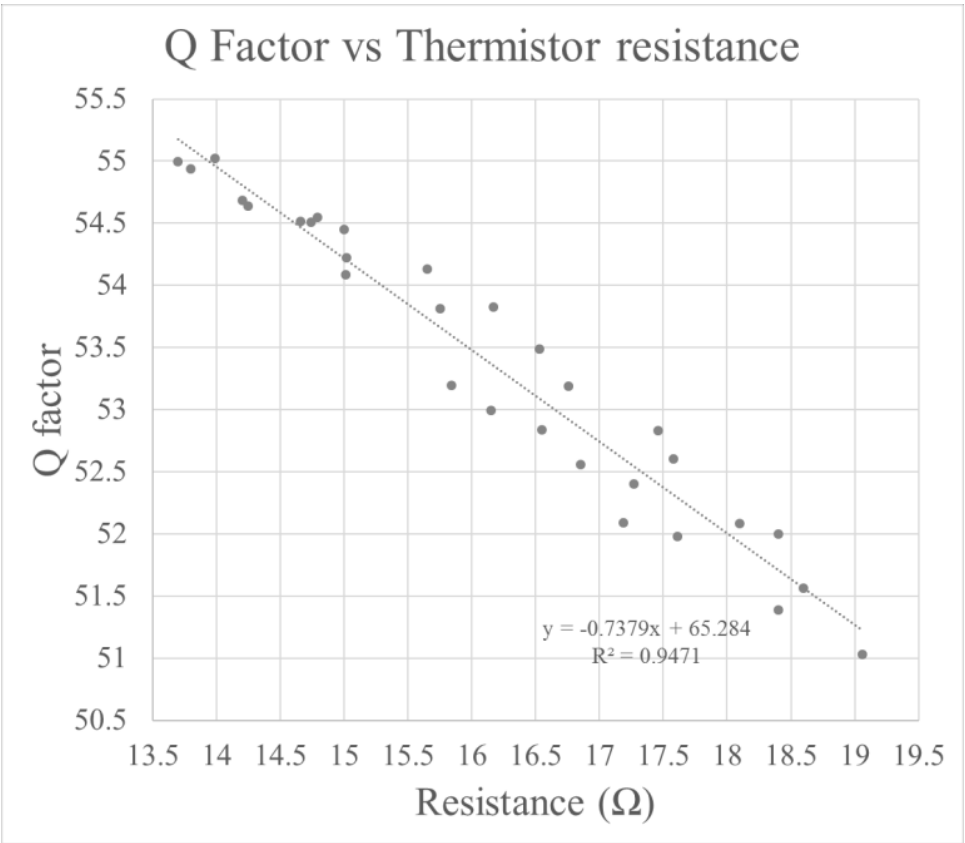

Figure 3.9 Q factor versus Thermistor resistance

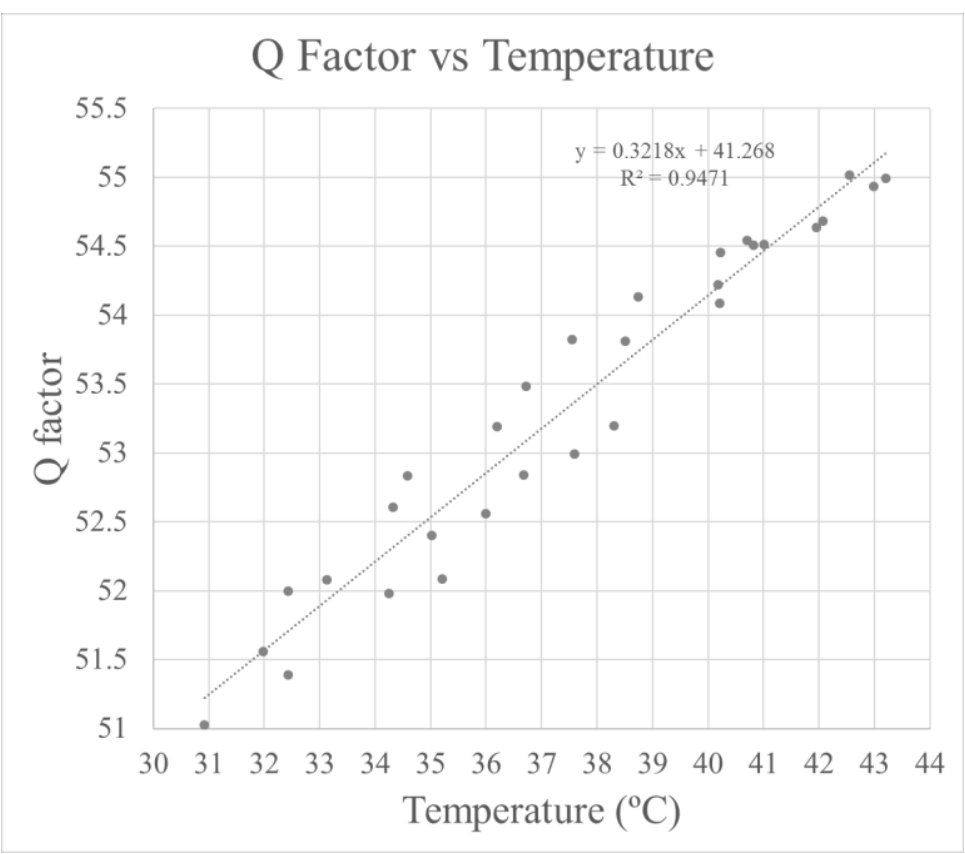

Figure 3.10 Q factor versus Temperature,

The sensitivity of the sensor was 0.3218 (Q factor) per ${ }^{\circ} \mathrm{C}$, calculated from Figure 3.10. The resolution of temperature depends on the characteristics of the detection system 
used. Although currently used detection system is functional, it is too costly and bulky for commercial applications.

\subsubsection{Repeatability}

Repeatability test was conducted for continues heating and cooling cycles. The Q factor and temperature was plotted against time. The temperature was varied between 35 ${ }^{\circ} \mathrm{C}$ and $40{ }^{\circ} \mathrm{C}$. Figure 3.11 shows the stability of the sensor response for the repeatability test Repeatability test can be considered as a measure for accuracy and precision of the system. The accuracy of the sensor is between $-0.44741{ }^{\circ} \mathrm{C}$ and $-2.07255{ }^{\circ} \mathrm{C}$ and the precision is between $\pm 0.40160{ }^{\circ} \mathrm{C}$ and $\pm 0.73252^{\circ} \mathrm{C}$.

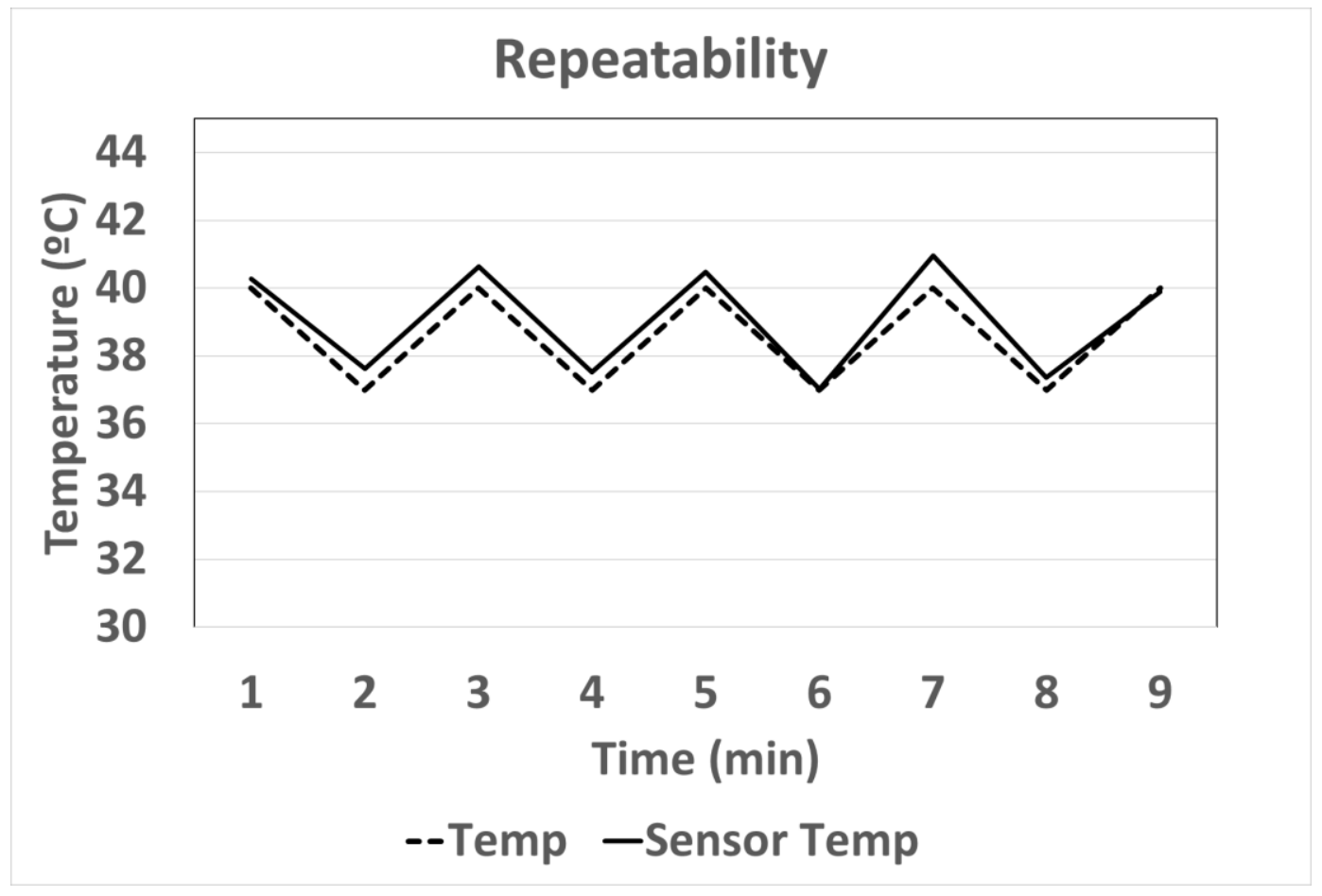

Figure 3.11 Repeatability test of the sensor at $35^{\circ} \mathrm{C}$ and $40^{\circ} \mathrm{C}$. 


\subsection{Results}

The results from the sensor performance show that sensitivity of the sensor is 0.3218 per $^{\circ} \mathrm{C}$, and by using a high precision detection system small temperature variation can be detected. In the study by Romano et al., [44], it was shown that a value of $0.9^{\circ} \mathrm{C}$ or less is sensitive enough to detect infection in the case of thermographic imaging. The resolution of the detection system should be at least half of this value, preferably $1 / 10^{\text {th }}$.

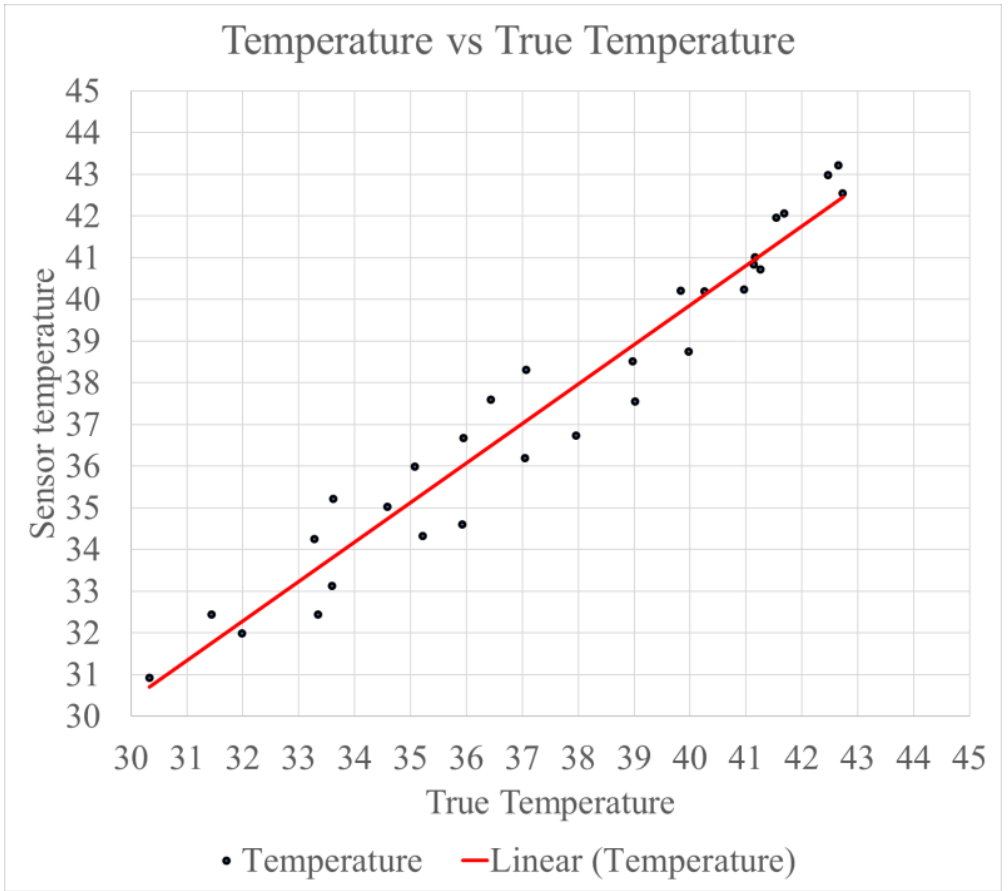

Figure 3.12 Temperature measured by sensor versus true temperature measured by the thermistor.

The temperature measurements shown in Figure 3.13 were collected when temperature was being increased or decreased. Since the measurements were not taken after the temperature was stable, an error was expected due to the small difference in heat transfer rates between the LC sensor and the LM35 temperature sensor. The error is reflected in the small offset of the linear calibration curve in the figure (the red line in 
Figure 3.12 is supposed to cross the lower left corner of the graph, but instead it has a 0.4 ${ }^{\circ} \mathrm{C}$ offset in the $y$-axis). This error is not expected when this technology is applied clinically. Since in practice, a single temperature measurement will be collected by averaging multiple measurements over a time period, any transients in temperature will be overcome by the averaging. Furthermore, we do not expect the body temperature to experience a high rate of temperature change (in the benchtop experiment, temperature change can be as high as $2{ }^{\circ} \mathrm{C} / \mathrm{min}$, but the temperature at the surgery site should be fairly stable within minutes), thus we do not expect the error in Figure 3.12 to play a significant role when the sensor is implemented.

The Relative Error (in \%) (Equation (3.17)) in temperature measurement of the sensor was $-0.0132 \% \pm 2.3559 \%$ from Figure 3.12 using the formulae

$$
\begin{aligned}
& \text { Relative Error } \% \\
& \qquad=\frac{\text { Temperature }- \text { True temperature }}{\text { True Temperature }} \times 100 \%
\end{aligned}
$$

There are several factors that can affect the error in the temperature measurement. Reducing such error can help improve the accuracy and precision of the sensor. Most important source of error for the sensor is from the experimental errors. There are several factors that can produce experimental errors such as errors due to the excitation delays between different measurements (resistance measurement and impedance measurement) and errors in calibration of the thermistor resistance and temperature. In thermistor 
resistance calibration, the sensor (LM35) [59] used has an accuracy of $\pm 0.1^{\circ} \mathrm{C}$. Higher precision integrated-circuit temperature sensor (like LMT70 precision of $\pm 0.05{ }^{\circ} \mathrm{C}$ ) [60] can be used to improve the calibration. The temperature also influences the inductance and capacitance. Ong et al., [52] developed a planar LC temperature sensor. In the planar LC temperature sensor, the temperature rise causes variation in the inductance and capacitance. The measure of resonant frequency shift corresponded to temperature. The temperature dependence of resonant frequency is small $\left(\sim 1 \mathrm{KHz}\right.$ per $\left.{ }^{\circ} \mathrm{C}\right)$, but further studies need to be conducted to study whether this frequency shift will affect the accuracy of sensor. Selfheating is another important phenomenon that can cause error in temperature measurements. The study does not quantify self-heating effect of the thermistor [46]. Characterizing the thermistor self-heating when powered wirelessly will also help to reduce the error. Designing a different detection system with 2 different coils, for excitation and detection, can increase the detection range and sensor response.

\subsection{Sensor performance in actual tissue}

All experiments were conducted in air - both the coil and sensor were not immersed or embedded in a medium that simulating the actual tissue. To confirm that the performance of the sensor is consistent when it is embedded inside body tissue, the response of the sensor was measured again when a medical grade ballistic gelatin (from Clear Ballistics) was used to separate the sensor and the coil. Marchal et al., in 1989 [61] showed that gelatin based phantoms can mimic the dielectric properties of tissue. According to Figure 3.13, 
there is no considerable difference between the $\mathrm{Q}$ factor test done in air and with gelatin in between the sensor and detection coil.

This result indicates that the sensor should perform similarly when the sensorized screw is implanted in the human body. It has to note that the reason that the sensor signal did not experience significant decrease was due to the relatively low frequency of this sensor, which was less than $30 \mathrm{MHz}$. At higher frequencies, such as $300 \mathrm{MHz}$ and above, electromagnetic radiation absorption is higher in tissue. This can result in weaker sensor performance in range of $300 \mathrm{MHz}-3 \mathrm{GHz}$.

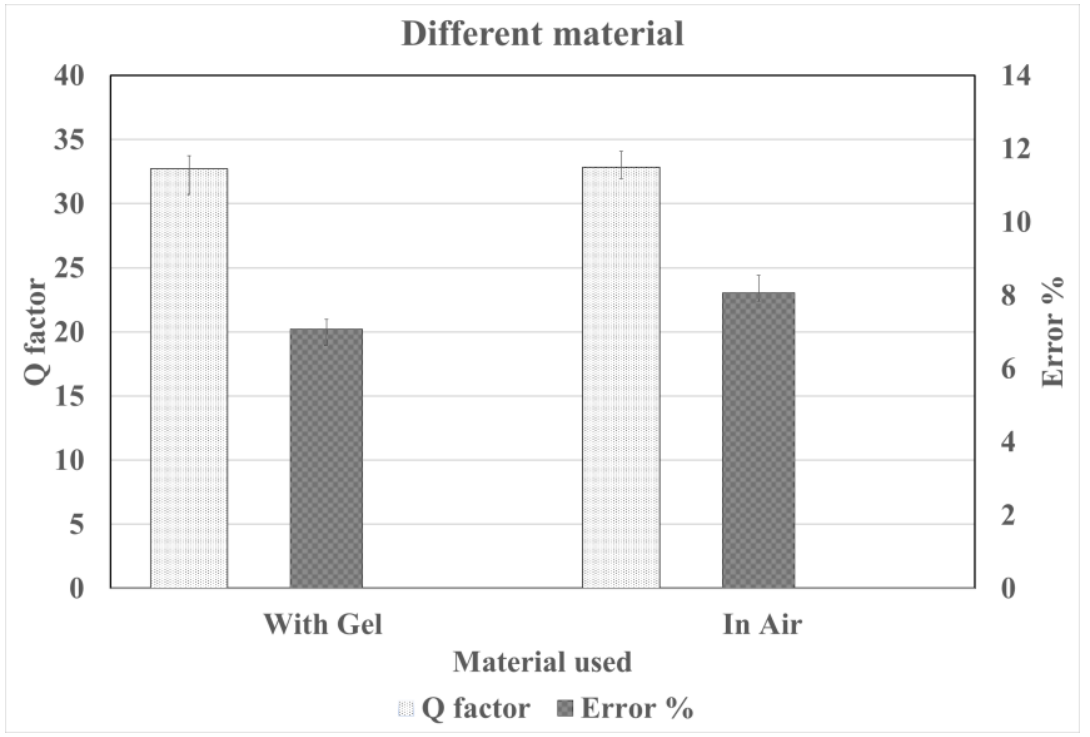

Figure 3.13 Difference in sensor performance with materials.

The measurement was done at $30 \mathrm{~mm}$ separation between the coil and sensor, with gelatin or air as the separating medium. 


\section{Conclusion}

In recent years, wireless sensor technology has advanced with the introduction cheap efficient fabrication technologies. RFID tags are being used widely to monitor different consumer goods. A similar technology called near field communication (NFC) is being used by in smartphones for different application including cashless payments. Such remote query systems enable easier and faster detection than wired connections. The aim of this project is to develop a wireless system, similar to the abovementioned technologies but passive, on an orthopedic implant with an incorporated temperature sensor to monitor the infections at the site of implantation. In this project, a temperature sensor was embedded into an interference screw. This temperature sensor can be used for detecting the infection at the site of screw implantation. This is based on the hypothesis that infection can cause local temperature rise around the implanted screw. In this project, several experiments were conducted to optimize the design for use in knee ligament reconstruction (MCL and LCL). A detection coil was also designed and optimized for this specific application. During the testing of coil, it was noted that the coil diameter depended on the depth of sensor. The diameter can be computed for Equation (2.15). The experiment was also able to demonstrate how coil wire gauge, number of windings and power effect the sensor response.

A major advantage of this sensor is its improvement for patient comfort. This sensor can allow short term monitoring in the hospital, as well as and long term monitoring at home considering the fact that the infections can occur up to 2 years after the surgery. For the latter application, the detection device can be made small and portable enough to be 
used by the patient. Unlike catheter type sensors, this system is not invasive and does not increase the risk of infection or implant failure. Similarly, there is no additional intrusive procedure introduce by the system that may cause patient discomfort. With this technology, the patient can still move around, and undergo rehabilitation and therapy with the sensor is implanted.

The factors influencing the performance were also identified and investigated. The induction winding of the sensor plays an important role in the performance of the sensor. Higher number of windings and wider diameter wires produce better coupling between the sensor and the detection coil. The sensor performance was also analyzed for different spatial positions relative to the detection coil.

The sensors temperature sensitivity was calculated to be around 0.322 per ${ }^{\circ} \mathrm{C}$. A relative error of $-0.0132 \% \pm 2.36 \%$ was calculated form the calibrated temperature readings. It was shown in a clinical trial by Romano et al., [44] that $0.9^{\circ} \mathrm{C}$ of differential temperature measurement on the skin surface shows an infection at the site of a surgery. The current detection system is costly and bulky for commercial application. Hence, a cheaper and smaller detection system needs to be designed. The resolution of such a detection system needs to be low enough to monitor temperatures as low as $0.05{ }^{\circ} \mathrm{C}$.

The temperature sensor can produce more accurate and precise measurements by quantifying different source of errors. More characterization needs to be carried out to study the self-heating effects in the thermistor when it is being wirelessly powered. Temperature dependent resonant frequency shift is another phenomenon observed in the 
sensor. Studies need to be done to characterize this frequency shift and the error introduced by it.

HP Schwan et al., in 1971 [61][62] has shown that localized heating can be produced due to the electromagnetic radiations. This heating effect is mostly dependent on the frequency of electromagnetic wave applied on the tissue. Microwave frequencies (300 $\mathrm{MHz}-300 \mathrm{GHz}$ ) produce considerably higher heating in tissue than lower frequency radio waves $(300 \mathrm{MHz}-3 \mathrm{kHz})$. Since the frequency range of sensor detection is $15-30 \mathrm{MHz}$, the detection coil does not produce considerably high level of heating. Prolonged exposure at lower frequency also may cause heating. In this experiment, heating effects are minimum due to the fact that only short time $(\sim 2 s)$ exposure of radiations are needed for measuring the temperature of the sensor. The data can be interrogated within a period of 5 minutes (with multiple signal bursts of $\sim 2 \mathrm{~s}$ ) for data averaging to reduce error. This helps to reduce the effective radiation exposure of the tissue and alleviate any heating due to radiation at the site of implant. It also should be noted that continuous monitoring is not required since a maximum of 4 measurements are only required in a day. During interrogation, although electrical current induced in the sensor may cause the thermistor to heat up, the heating is not expected to be significant due to the low power and short interrogation time. 


\section{Reference}

1. Whitehouse, J.D., et al., The impact of surgical-site infections following orthopedic surgery at a community hospital and a university hospital: Adverse quality of life, excess length of stay, and extra cost. Infection Control and Hospital Epidemiology, 2002. 23(4): p. 183-189.

2. Horan, T.C., et al., CDC definitions of nosocomial surgical site infections, 1992: a modification of CDC definitions of surgical wound infections. American journal of infection control, 1992. 20(5): p. 271-274.

3. Klevens, R.M., et al., Estimating health care-associated infections and deaths in U.S. hospitals, 2002. Public Health Rep, 2007. 122(2): p. 160-6.

4. G. Nakagami PhD, R., et al., Predicting delayed pressure ulcer healing using thermography: a prospective cohort study. Journal of Wound Care, 2010. 19(11): p. 465472.

5. Venta, I., et al., Thermographic imaging of postoperative inflammation modified by anti-inflammatory pretreatment. J Oral Maxillofac Surg, 2001. 59(2): p. 145-8; discussion 149-50.

6. Christensen, J., et al., Thermography as a quantitative imaging method for assessing postoperative inflammation. Dentomaxillofac Radiol, 2012. 41(6): p. 494-9.

7. Romanelli, M., et al., Use of diagnostics in wound management. Curr Opin Support Palliat Care, 2013. 7(1): p. 106-10. 
8. Mufti, A., P. Coutts, and R.G. Sibbald, Validation of Commercially Available Infrared Thermometers for Measuring Skin Surface Temperature Associated with Deep and Surrounding Wound Infection. Advances in Skin \& Wound Care, 2015. 28(1): p. 1116.

9. Kurtz, S.M., et al., Economic burden of periprosthetic joint infection in the United States. J Arthroplasty, 2012. 27(8 Suppl): p. 61-5 e1.

10. Zimmerli, W., Clinical presentation and treatment of orthopaedic implantassociated infection. J Intern Med, 2014. 276(2): p. 111-9.

11. Sendi, P., et al., Clinical comparison between exogenous and haematogenous periprosthetic joint infections caused by Staphylococcus aureus. Clinical Microbiology and Infection, 2011. 17(7): p. 1098-1100.

12. Zimmerli, W., A. Trampuz, and P.E. Ochsner, Prosthetic-joint infections. New England Journal of Medicine, 2004. 351(16): p. 1645-1654.

13. Pandey, R., et al., Histological and microbiological findings in non-infected and infected revision arthroplasty tissues. Archives of orthopaedic and trauma surgery, 2000. 120(10): p. 570-574.

14. Steckelberg, J.M. and D.R. Osmon, Prosthetic joint infections, in Infections Associated with Indwelling Medical Devices, Third Edition. 2000, American Society of Microbiology. p. 173-209.

15. Zimmerli, W. and C. Moser, Pathogenesis and treatment concepts of orthopaedic biofilm infections. FEMS Immunology \& Medical Microbiology, 2012. 65(2): p. 158-168. 
16. Campoccia, D., L. Montanaro, and C.R. Arciola, The significance of infection related to orthopedic devices and issues of antibiotic resistance. Biomaterials, 2006. 27(11): p. 2331-2339.

17. Fink, B., et al., The value of synovial biopsy, joint aspiration and C-reactive protein in the diagnosis of late peri-prosthetic infection of total knee replacements. Bone \& Joint Journal, 2008. 90(7): p. 874-878.

18. Palestro, C.J., et al., Combined Labeled Leukocyte and Technetium 99m Sulfur Colloid Bone Marrow Imaging for Diagnosing Musculoskeletal Infection 1. Radiographics, 2006. 26(3): p. 859-870.

19. Widmer, A.F., New developments in diagnosis and treatment of infection in orthopedic implants. 2001, Oxford University Press.

20. Adirim, T.A. and T.L. Cheng, Overview of injuries in the young athlete. Sports Medicine, 2003. 33(1): p. 75-81.

21. Griffin, L.Y., et al., Noncontact anterior cruciate ligament injuries: risk factors and prevention strategies. Journal of the American Academy of Orthopaedic Surgeons, 2000. 8(3): p. 141-150.

22. Amiel, D., et al., Tendons and ligaments: a morphological and biochemical comparison. Journal of Orthopaedic Research, 1983. 1(3): p. 257-265.

23. Woo, S., et al., Measurement of mechanical properties of ligament substance from a bone-ligament-bone preparation. Journal of Orthopaedic Research, 1983. 1(1): p. 22-29. 
24. Race, A. and A.A. Amis, The mechanical properties of the two bundles of the human posterior cruciate ligament. Journal of biomechanics, 1994. 27(1): p. 13-24.

25. Provenzano, P., et al., Nonlinear ligament viscoelasticity. Annals of biomedical engineering, 2001. 29(10): p. 908-914.

26. Rees, J., A. Wilson, and R. Wolman, Current concepts in the management of tendon disorders. Rheumatology, 2006. 45(5): p. 508-521.

27. Woo, S.L.-Y., et al., Biomechanics of knee ligaments: injury, healing, and repair. Journal of biomechanics, 2006. 39(1): p. 1-20.

28. Järvinen, T.A., et al., Achilles tendon disorders: etiology and epidemiology. Foot and ankle clinics, 2005. 10(2): p. 255-266.

29. Thornton, G., et al., Biomechanics of the Musculo-Skeletal System. 2007, John Wiley \& Sons, England.

30. Schultz, R.A., et al., Mechanoreceptors in human cruciate ligaments. A histological study. J Bone Joint Surg Am, 1984. 66(7): p. 1072-1076.

31. Leadbetter, W. and B. Wayne, Soft tissue athletic injury. Sports injuries: mechanisms, prevention, treatment. Baltimore: Williams \& Wilkins, 1994: p. 736-7.

32. Whiting, W.C. and R.F. Zernicke, Biomechanics of musculoskeletal injury. 2008: Human Kinetics.

33. Frank, C., et al., Normal ligament properties and ligament healing. Clinical orthopaedics and related research, 1985. 196: p. 15-25. 
34. Frank, C., Ligament injuries: pathophysiology and healing. Athletic injuries and rehabilitation, 1996: p. 9-26.

35. Oakes, B., The classification of injuries and mechanisms of injury, repair and healing. Textbook of science and medicine in sport. Melbourne: Blackwell Scientific Publications, 1992: p. 200-17.

36. Ng, G.Y., et al., The long-term biomechanical and viscoelastic performance of repairing anterior cruciate ligament after hemitransection injury in a goat model. The American journal of sports medicine, 1996. 24(1): p. 109-117.

37. Widmaier, E.P., H. Raff, and K.T. Strang, Vander's human physiology. 2006: McGraw Hill Boston, Mass, USA.

38. Harner, C.D., et al., Quantitative analysis of human cruciate ligament insertions. Arthroscopy: The Journal of Arthroscopic \& Related Surgery, 1999. 15(7): p. 741-749.

39. Wright, R.W., et al., Ipsilateral graft and contralateral ACL rupture at five years or more following ACL reconstruction. J Bone Joint Surg Am, 2011. 93(12): p. 1159-1165.

40. Groves, E.H., OPERATION FOR THE REPAIR OF THE CRUCIAL LIGAMENTS. The Lancet, 1917. 190(4914): p. 674-676.

41. Wasilewski, S.A., D.J. Covall, and S. Cohen, Effect of surgical timing on recovery and associated injuries after anterior cruciate ligament reconstruction. The American journal of sports medicine, 1993. 21(3): p. 338-342. 
42. Fierheller, M. and G. Sibbald, A Clinical Investigation into the Relationship between Increased Periwound Skin Temperature and Local Wound Infection in Patients with Chronic Leg Ulcers. Advances in Skin \& Wound Care, 2010. 23(8): p. 369-378.

43. Epari, D.R., et al., Pressure, oxygen tension and temperature in the periosteal callus during bone healing —an in vivo study in sheep. Bone, 2008. 43(4): p. 734-739.

44. Romanò, C.L., et al., Value of digital telethermography for the diagnosis of septic knee prosthesis: a prospective cohort study. BMC musculoskeletal disorders, 2013. 14(1): p. 7.

45. Romano, C.L., et al., Healing of surgical site after total hip and knee replacements show similar telethermographic patterns. Journal of orthopaedics and traumatology, 2011. 12(2): p. $81-86$.

46. Fraden, J., Temperature Sensors. 2016: p. 585-643.

47. DeRouin, A.J., et al., A Wireless Inductive-Capacitive Resonant Circuit Sensor Array for Force Monitoring. Journal of Sensor Technology, 2013. 03(03): p. 63-69.

48. DeRouin, A.J., et al., Development and Application of the Single-Spiral InductiveCapacitive Resonant Circuit Sensor for Wireless, Real-Time Characterization of Moisture in Sand. Journal of Sensors, 2013. 2013: p. 1-7.

49. Ong, K.G. and C.A. Grimes, A resonant printed-circuit sensor for remote query monitoring of environmental parameters. 2000. 
50. Ong, K.G., et al., Monitoring of bacteria growth using a wireless, remote query resonant-circuit sensor: application to environmental sensing. Biosensors \& Bioelectronics, 2001. 16(4-5): p. 305-312.

51. Ong, K.G., K.F. Zeng, and C.A. Grimes, A Wireless, Passive Carbon NanotubeBased Gas Sensor. Ieee Sensors Journal, 2002. 2(2): p. 82-88.

52. Ong, K.G., et al., Design and application of a wireless, passive, resonant-circuit environmental monitoring sensor. Sensors and Actuators A: Physical, 2001. 93(1): p. 3343.

53. Lee, Y., RFID coil design. Microchip Technol. Inc. Appl. Note, 1998.

54. Landt, J., The history of RFID. IEEE potentials, 2005. 24(4): p. 8-11.

55. Want, R., An introduction to RFID technology. IEEE pervasive computing, 2006. 5(1): p. 25-33.

56. Schmieding, R., Interference screw and cannulated sheath for endosteal fixation of ligaments. 1993, Google Patents.

57. Boileau, P., et al., Arthroscopic biceps tenodesis: a new technique using bioabsorbable interference screw fixation. Arthroscopy: The Journal of Arthroscopic \& Related Surgery, 2002. 18(9): p. 1002-1012.

58. Hehl, F.W. and Y.N. Obukhov, Foundations of classical electrodynamics: Charge, flux, and metric. Vol. 33. 2012: Springer Science \& Business Media. 
59. Instruments, T., LM35 Precision Centigrade Temperature Sensors. LM35 datasheet, Aug, 1999.

60. Instruments, T., Wearable Temperature Sensing Layout Considerations Optimized for Thermal Response. LMT70 Application Report (SNIA021).

61. Dielectric peoperties of gelatine phantoms used for simulation of biological tissues between 10 and $50 \mathrm{MHz}$. Hyperthermia, 1989.

62. Schwan, H.P., Interaction of microwave and radio frequency radiation with biological systems. IEEE Transactions on microwave theory and techniques, 1971. 16(2): p. $146-152$. 